\title{
Successive Interference Cancellation and Fractional Frequency Reuse For LTE Uplink Communications
}

\author{
Jianhua He, Zuoyin Tang, Zhiguo Ding and Dapeng Wu
}

\begin{abstract}
Cellular networks are increasingly densified to deal with the fast growing wireless traffic. Interference mitigation plays a key role for the dense cellular networks. Successive interference cancellation (SIC) and fractional frequency reuse (FFR) are two representative inter-cell interference (ICI) mitigation techniques. In this paper we study the application of both SIC and FFR for LTE uplink networks, and develop an analytical model to investigate their interactions and impact on network performance. The performance gains with FFR and SIC are related to key system functionalities and variables, such as SIC parameters, FFR bandwidth partition, uplink power control and sector antennas. The ICIs from individual cell sectors are approximated by log-normal random variables, which enables low complexity computation of the aggregate ICI with FFR and SIC. Then network performance of site throughput and outage probability is computed. The model is fast and has small modelling deviation, which is validated by simulations. Numerical results show that both SIC and FFR can largely improve network performance, but SIC has an impact over FFR. In addition, most of the network performance gains with SIC could be obtained with a small number of SIC stages applied to a few sectors.
\end{abstract}

Keyworks: Long-term Evolution (LTE); System Performance Modeling; Successive Interference Cancellation; Fractional Frequency Reuse.

\section{INTRODUCTION}

D UE to the fast growing wireless traffic there is a high pressure on cellular network operators to look ahead to find new capacity scaling methods to meet the demands of the future 5G standard [1]-[3]. There are many system architectures and radio techniques under extensive research to increase network capacity, including massive and multiuser MIMO, 3D beamforming, millimetre wave transmission and non-orthogonal multiple access and network densification. Among these approaches network densitification with small cells and heterogeneous networks is a widely accepted and economical one [1]-[3].

With increasingly dense and interference limited cellular networks interference mitigation technologies will play a key part in improving network performances [4]. Successive interference cancellation (SIC) and Fractional frequency reuse (FFR) are two representative inter-cell interference mitigation techniques [4]-[6]. With FFR cells are partitioned into

Jianhua He is with School of Information Science and Engineering, East China University of Science and Technology, China, and School of Engineering and Applied Science, Aston University, UK. Email j.he7easton.ac.uk. Zuoyin Tang is with School of Engineering and Applied Science, Aston University, UK. Email: z.tang1@aston.ac.uk. Zhiguo Ding is with School of Computing and Communications Lancaster University, UK. Email: z.ding@lancaster.ac.uk. Dapeng Wu is with Department of Electrical and Computer Engineering, University of Florida. Email: dpwu@ufl.edu. Corresponding Author: Zuoyin Tang. zones where different frequency reuse factors are used. In the literature the application of FFR to cellular networks in the downlink communications has been widely studied [6][8]. On the other hand, the idea of SIC is to decode the signals according to certain order and subtract the decoded signals from the aggregate received signal successively [5]. An extensive survey on the SIC for orthogonal frequency division multiplexing (OFDM) systems with single and multiple antennas was presented in [5], with a focus on the bit error ratio (BER) performance and complexity tradeoff analysis.

In the literature the research on SIC was focused on the physical layer communications aspect [5]. Recently there are increasing interests in the application of SIC to cellular networks and exploring the network level performance gains that can be brought by SIC. Two representative applications are the full duplex technology and the non-orthogonal frequency division multiplexing (NOMA) in downlink communications for the 5G system [1], [9]-[12]. In the NOMA based networks the same frequency resources are scheduled to multiple users and SIC is applied at the receivers to cancel intra-cell multiple user interference. However due to the use of uplink power control there is very little space left for NOMA to perform intra-cell cancellation in the uplink communications. A few works studied the application of SIC to inter-cell cancellation in cellular uplink communications [14], [16].

It is noted that FFR applies frequency reuse for interference mitigation at the cost of lower frequency resource utilization, while SIC attempts to cancel interferences and encourage universal frequency reuse. The interaction of these two interference mitigation technology is interesting, but has not been revealed in the literature. While the performance of joint FFR and SIC can be evaluated by simulation approach [8], [20], [21], fast and reliable evaluation with analytical approach is still an open reserach issue. In this paper we investigate the application of both SIC and FFR for ICI mitigation in LTE uplink communications. We assess the performance gain of SIC, and check if it can be achieved while the signal overhead and SIC processing delay are controlled to tolerable levels. An analytical model is developed for fast and accurate evaluation of FFR and SIC technologies for LTE uplink communications, and identification of their interaction and impact.

\section{A. Related Work}

a) General models for uplink ICI: Uplink ICI modeling for cellular networks (especially OFDM based networks) is a subject receiving strong research interests. A widely used model for the uplink ICI is Wyner model [25], in which ICI 
is assumed as a weight of the aggregate signals transmitted from adjacent cells but the ICI value is left to be determined. With given site locations and user associations to the eNodeBs, the moments of single cell uplink ICI can be computed and are explored to compute aggregate uplink ICI to the target eNodeB. Haas and McLaughlin derived probability distribution function (PDF) of adjacent channel uplink interference from single cell of cellular systems [26]. Similarly Zhu et al derived PDF of single cell uplink ICI with power control in OFDMA networks [27]. It is noted that aggregate ICI expression was not derived from the PDF of single cell uplink ICI [26] [27], and network performance was not analyzed. Elayoubi et al studied the uplink ICI and capacity in 3G LTE systems without shadowing [28]. Karray studied uplink resource allocation and network performance in both CDMA and OFDMA networks [29]. A framework of modelling uplink ICI was reported with scheduling in [30] and with power compensation schemes in [31]. The model considers one tier of regularly laid interfering cells, and generalized K-composite fading was assumed for analytical model tractability. Singh et al developed a momentmatched log-normal modeling of uplink ICI with power control and shadowing for CDMA system [32]. The aggregate uplink ICI is assumed to be log-normal distributed. The moment-matched approach was applied to OFDMA networks with sector antennas for networks without shadow fading [34]. Norlan et al applied the stochastic geometric tool to model OFDMA uplink ICI and network performances [24]. The model was extended by ElSawy et al for cellular uplink transmission with truncated power control [35]. It is noted that, while stochastic geometry tool is powerful for performance analysis of cellular networks [22], the above stochastic models are not directly applicable to practical cellular network with sector antennas and given network layouts.

b) Analytical models for cellular networks with FFR and SIC: While there are efficient analytical models available for FFR application in the downlink direction of cellular networks [6]-[8], only a few models were proposed for uplink performance evaluation with FFR [33] [36]. In the analytical model [36] sector antenna is taken into account but fading was not considered. On the other hand, there are increasing research interests in using stochastic geometry tools to model network performance with SIC [13], [14], [16], [18]. Weber et al presented upper and lower bounds of outage probability for wireless ad hoc networks with SIC in [13], where fading effcts were not considered. The work was extended by Zhang and Haenggi to model the bounds on the outage probability with SIC and fading effects [14] and throughput [15]. Wildemeersch et al derived expressions of the success probability to cancel the $k$ th strongest signal and coverage probability of uplink communications with SIC [16]. Energy efficiency of SIC was analyzed in [17]. Downlink coverage probability was derived for Poisson networks using factorial moment measures [18]. Ma et al analyzed the SIC performance of device to device enabled cellular networks [19].

\section{B. Main Contributions and Organization}

In the existing works sector antennas were not considered except [34]. In addition there is no analytical model that can predict system throughput gains with SIC and FFR for uplink communications. In this paper we extend our previous works on modeling uplink ICI [23], to develop a unified and fast analytical model for LTE uplink communications with SIC and FFR interference mitigation technologies. The analytical model is then applied to evaluate their interactions and network performance gains and the impact of relevant parameters related to FFR and SIC.

The main research work and contributions of this paper are summarised from three aspects: 1) A general analytical model is proposed for performance prediction of LTE uplink communication with SIC and FFR. Fractional power control, shadow fading, sector antenna radiation patterns and irregular cellular layouts are taken into account. In the model single sector uplink ICIs are approximated by log-normal distributions, which enables fast and accurate computation of aggregate ICI and other performance metrics of interest. The proposed model is validated by simulations. 2) We develop an analytical approach to model a general abstracted SIC process with key SIC parameters (number of SIC stages, number of sectors for SIC and SIC decoding threshold). The SIC process model is a key part of the overall analytical model for network performance prediction. The analytical model for the SIC process enables accurate and fast computation of SIC decoding success probability, and evaluation of the aggregate ICI and network performance with SIC. 3) With both analytical and simulation approaches, which provide cross verification and higher reliability, performance evaluation of LTE networks with FFR and SIC is conducted. Numerical results show that SIC has larger potential even when it is applied to only a few sectors with a few SIC stages. The results also reveal interesting insights to the interaction and impact of SIC and FFR.

The rest of this paper is organised as follows. In Section II system model and assumptions are introduced. In Section III, the overall analytical framework and the single sector ICI model with FFR are presented. Section IV presents the SIC process, the sector and aggregate ICI models with SIC. Section V presents the analysis of network performance with FFR and SIC, in terms of SINR, site throughput and outage probability. In Section VI numerical results and discussions are presented. Section VII concludes the paper.

\section{SySTEM MODEL}

\section{A. Network Layout and Resource Allocation}

We consider LTE networks with $N_{\text {sites }}$ eNodeB sites and 3 sectors per site. The sites are labeled from 1 to $N_{\text {sites }}$, ranked in ascending order of distance from site 1. Without loss of generality eNodeB 1 is set as the target site and located at the origin $(0,0)$. The inter-site distance is denoted by $R_{\mathrm{ISD}}$.

The total number of sectors (denoted by $N_{\text {sect }}$ ) is $3 N_{\text {sites }}$. The $j$ th sector of the $n$th site is denoted by $\mathcal{A}_{n, j}$, where $n \in\left[1, N_{\text {sites }}\right]$. For alleviation of notation, sector $\mathcal{A}_{n, j}$ and its sector antenna (SA) are also labeled by $k$, with $k=$ $3(n-1)+j, n \in\left[1, N_{\text {sites }}\right], j \in[1,3]$, and $k \in\left[1, N_{\text {sect }}\right]$. An example of network layout with one tier of interfering eNodeBs is presented in Fig. 1(a). 


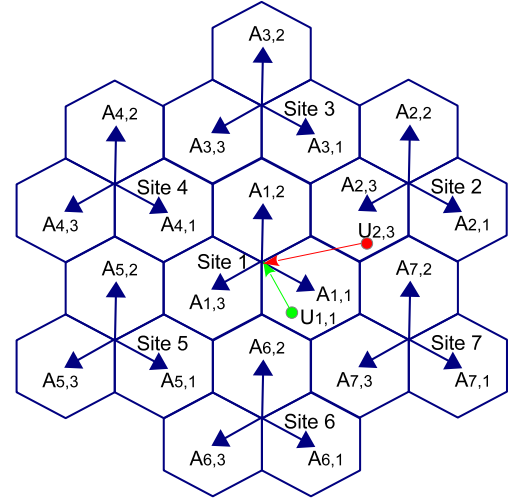

(a) Network layout with 3-sectors.

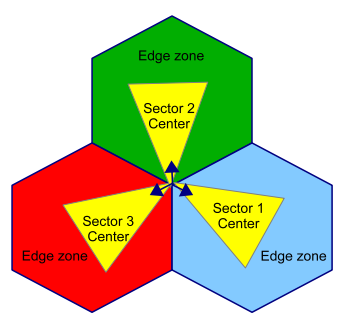

(b) FFR sector partition to center and edge zones.

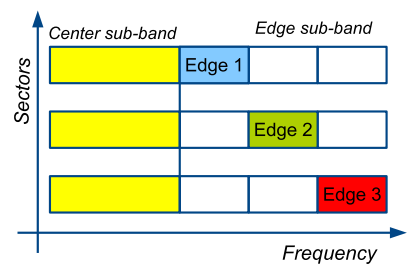

(c) FFR bandwidth partition.
Fig. 1. Illustration of a) cellular network layout with 3-sectors with a target user $U_{1,1}$ in sector $\mathcal{A}_{1,1}$ and an interfering user from sector $\mathcal{A}_{2,3}$; b) FFR sector partition into center and edge zones; c) FFR bandwidth partition for center and edge zones.

In LTE networks single carrier frequency division multiple access (SC-FDMA) is chosen for uplink multiple access. Spectrum resources are split into a number of orthogonal narrowband sub-carriers with a space of $15 \mathrm{kHz}$ and organised into resource blocks for allocation [2]. The minimum resource for allocation has $1 \mathrm{~ms}$ in time domain and $180 \mathrm{kHz}$ in frequency domain [2]. For ease of notation, we define a physical resource block (PRB) as the minimum resource used for transmission with $180 \mathrm{kHz}$ by one slot. We assume a round-robin scheduler for resource allocation. A fully loaded network is assumed, in which all the available resource blocks are allocated to the users in the sectors. Users are uniformly distributed in the network.

Table 1 lists the main notations used in this paper.

\section{B. Channel Model, Antenna Model and Power Control}

Without loss of generality, we can focus our study on the performances of users located in sector 1 with one PRB. Let us consider a general user $u_{k}$ served by sector $k$, where $k \in$ $\left[1, N_{\text {sect }}\right]$. Define the signal power $\mathcal{P}_{n, u_{k}}$ received by sector $n$ from a user $u_{k}$ of sector $k$, which is expressed by:

$$
\mathcal{P}_{n, u_{k}}=P_{u_{k}}^{\mathrm{t}} G_{\mathrm{PL}}\left(n, u_{k}\right) G_{\mathrm{A}}\left(n, u_{k}\right) \psi_{n, u_{k}}
$$

where $P_{u_{k}}^{\mathrm{t}}$ is the transmit power from user $u_{k}$ over a PRB, $G_{\mathrm{PL}}\left(n, u_{k}\right)$ is the path loss between the site of sector $n$ and user $u_{k}, G_{\mathrm{A}}\left(n, u_{k}\right)$ is the antenna gain between sector $n$ and user $u_{k}$, and $\psi_{n, u_{k}}$ is the shadow fading between sector $n$ and user $u_{k}$. We let $P_{n, u_{k}}^{\mathrm{r}}$ denote the part of received power by sector $n$ from user $u_{k}$ without shadowing:

$$
P_{n, u_{k}}^{\mathrm{r}}=P_{u_{k}}^{\mathrm{t}} G_{\mathrm{PL}}\left(n, u_{k}\right) G_{\mathrm{A}}\left(n, u_{k}\right) .
$$

The path loss model specified in [37] for outdoor line-ofsight communications is used:

$$
G_{\mathrm{PL}}(d)=-34.02-22 \log _{10}(d)[\mathrm{dB}],
$$

where $d$ is the distance between a considered pair of an eNodeB site and a user.

Radiation pattern with antenna gain $G_{\mathrm{A}, \mathrm{h}, \mathrm{v}}(\vartheta, \theta)$ given by [37] is used:

$$
\begin{aligned}
& G_{\mathrm{A}, \mathrm{h}, \mathrm{v}}^{\mathrm{dB}}(\vartheta, \theta)=-\min \left(-\left(G_{\mathrm{A}, \mathrm{h}}^{\mathrm{dB}}(\vartheta)+G_{\mathrm{A}, \mathrm{v}}^{\mathrm{dB}}(\theta)\right), G_{\mathrm{Front}}^{\mathrm{dB}}\right) \\
& G_{\mathrm{A}, \mathrm{h}, \mathrm{v}}(\vartheta, \theta)=10^{G_{\mathrm{A}, \mathrm{h}, \mathrm{v}}^{\mathrm{dB}}(\vartheta, \theta) / 10}
\end{aligned}
$$

where $G_{\mathrm{A}, \mathrm{h}}(\vartheta)$ and $G_{\mathrm{A}, \mathrm{v}}(\theta)$ are the normalized horizontal and vertical radiation pattern offset of the considered sector antenna, and $G_{\text {Front }}^{\mathrm{dB}}$ is the antenna front to back ratio.

$G_{\mathrm{A}, \mathrm{h}}(\vartheta)$ and $G_{\mathrm{A}, \mathrm{v}}(\theta)$ are approximated by [37]:

$$
G_{\mathrm{A}, \mathrm{h}}^{\mathrm{dB}}(\vartheta)=-\min \left(12\left(\frac{\vartheta}{\vartheta_{3 \mathrm{~dB}}}\right)^{2}, 25\right),
$$

and

$$
G_{\mathrm{A}, \mathrm{v}}^{\mathrm{dB}}(\theta)=-\min \left(12\left(\frac{\theta-\theta_{\mathrm{down}}}{\theta_{3 \mathrm{~dB}}}\right)^{2}, 20\right),
$$

where $\vartheta_{3 \mathrm{~dB}}$ and $\theta_{3 \mathrm{~dB}}$ are the horizontal and vertical halfpower beamwidth (HPBW), and $\theta_{\text {down }}$ is the down-tilt angle.

The shadow fading $\psi_{n, u_{k}}$ between general sector $n$ and user $u_{k}$ is widely assumed to follow a log-normal distribution. The logarithm of the shadow fading is assumed to have a zero mean and a standard deviation of $\sigma_{\mathrm{w}}$. The shadow fading between the interfering users and the target sector is assumed to be uncorrelated. Therefore we use $\psi_{n}$ to represent the shadow fading between any interfering user $u_{n}$ of sector $n$ and sector 1.

Fractional power control (FPC) approved by 3GPP is used in this paper. The following formula is used for transmit power $P_{u_{n}}^{\mathrm{t}}$ from the user $u_{n}$ of sector $n$ :

$P_{u_{n}}^{\mathrm{t}}=\min \left(P_{\max }, P_{\text {target }} M\left[G_{\mathrm{PL}}\left(n, u_{n}\right) G_{\mathrm{A}}\left(n, u_{n}\right)\right]^{-\beta}\right) / M$,

where $\beta$ is the power compensation factor, taking values of 0 , 0.4 to 1 with a step of $0.1 ; M$ is the number of PRBs allocated to a user, which is set to 6 in this paper. $P_{\max }$ is the maximal uplink transmit power, and $P_{\text {target }}$ is the target receive power.

\section{Fractional Frequency Reuse}

In this paper we considered a strict FFR. The sectors are partitioned to center and edge zones, with $\mathcal{A}_{k}^{c}$ and $\mathcal{A}_{k}^{e}$ denoting the center zone and the edge zone of sector $\mathcal{A}_{k}$, respectively, for $k=\left[1, N_{\text {sect }}\right]$. Accordingly the available bandwidth (denoted as $W$ ) is divided into two sub-bands, denoted by $W_{\mathrm{c}}$ and $W_{\mathrm{e}}$ for sector center and edge users, respectively. We let $\alpha$ denote the bandwidth partition coefficient, defined as $\alpha=W_{\mathrm{c}} / W$. Sector center zone uses a frequency reuse factor of 1 , while sector edge zone uses a frequency reuse factor of 3. Fig. 1(b) and Fig. 1(c) illustrate FFR sector partition and bandwidth 
TABLE I

NOTATIONS

\begin{tabular}{|c|c|c|c|}
\hline Notation & Meaning & Notation & Meaning \\
\hline$N_{\text {site }}$ & number of sites & $R_{\mathrm{ISD}}$ & inter-site distance \\
\hline$N_{\mathrm{a}}$ & number of sectors per site & $N_{\text {sect }}$ & number of sectors in the network \\
\hline $\mathcal{A}_{n, j}$ & $j$ th sector of $n$th site $\left(j=1, \ldots, N_{\mathrm{a}}\right)$ & $\mathcal{A}_{k}$ & sector $k$ or $\mathcal{A}_{n, j}$, with $k=(n-1) N_{\mathrm{a}}+j$ \\
\hline $\mathcal{P}_{n, u_{k}}$ & receive power by sector $n$ from sector $k$ user & $P_{u_{n}}^{\mathrm{t}}$ & transmit power from sector $n$ user \\
\hline$P_{\max }$ & maximal transmit power & $P_{\text {target }}^{u_{n}}$ & target received power at eNodeB \\
\hline$P_{n, u_{k}}^{\mathrm{r}}$ & mean received power by sector $n$ from user $u_{k}$ & & uplink power compensation factor \\
\hline$G_{\mathrm{PL}}\left(n, u_{k}\right)$ & path loss between sector $n$ and user $u_{k}$ & $G_{\mathrm{A}}\left(n, u_{k}\right)$ & antenna gain between sector $n$ and user $u_{k}$ \\
\hline$G_{\mathrm{A}, \max }$ & maximum antenna gain & $G_{\mathrm{A}, \mathrm{h}, \mathrm{v}}(\vartheta, \theta)$ & antenna radiation pattern with $\vartheta, \theta$ \\
\hline$\vartheta_{3 \mathrm{~dB}}, \theta_{3 \mathrm{~dB}}$ & horizontal and vertical HPBW & $\delta^{2}$ & noise power \\
\hline$\psi_{n, u_{k}}$ & shadow fading between sector $n$ and $u_{k}$ & $\sigma_{\mathrm{w}}$ & shadowing standard deviation (std) \\
\hline$I_{n}\left(I_{z, n}\right)$ & sector $n$ (zone $z$ ) ICI without shadowing & $\bar{I}_{n}, \widehat{I}_{n}$ & mean and variance of $I_{n}$ \\
\hline$I_{\mathrm{w}, n}\left(I_{\mathrm{w}, z, n}\right)$ & sector $n$ (zone $z$ ) ICI wi & $\mu_{\mathrm{w}, n}, \sigma_{\mathrm{w}, n}$ & mean, std of logarithm of $I_{\mathrm{w}, n}$ \\
\hline$I_{\text {sum }}\left(I_{\text {sum }, z}\right)$ & aggregate ICI (zone $z$ ) received by sector 1 & $\bar{I}_{\text {sum }}, \widehat{I}_{\text {sum }}$ & mean, variance of $I_{\text {sum }}$ \\
\hline$\gamma_{\text {out }}$ & outage SINR threshold & $\mu_{I_{\text {sum }}}, \sigma_{I_{\text {sum }}}$ & mean, std of logarithm of $I_{\text {sum }}$ \\
\hline$\gamma_{z, u}$ & SINR of user $u$ in zone $z$ of sector 1 & $\bar{\gamma}_{z, u}, \widehat{\gamma}_{z, u}$ & mean, variance of SINR $\gamma_{z, u}$ \\
\hline$\mu_{z, u}, \sigma_{z, u}$ & mean, std of natural logarithm of $\gamma_{z, u}$ & $\gamma_{\text {net }, z}, \eta_{\text {site }}, \mathcal{O}_{\text {net }}$ & zone $z$ SINR, site throughput, outage prob. \\
\hline$N_{\text {stg }}$ & Number of SIC stages & $\epsilon$ & SIC decoding threshold \\
\hline$N_{\text {soi }}$ & Number of sector of interests (SOI) & $\Omega_{\text {soi }}$ & set of SOI \\
\hline$P_{\text {sic }, n, s}$ & prob. of sector $n$ ICI cancelled at stage $s$ & $P_{\text {sic }, s}$ & SIC success prob. at stage $s$ \\
\hline$I_{n, s}$ & residual ICI from sector $n$ at end of stage $s$ & $I_{\mathrm{sic}, n, s}$ & sum of noise, agg. residual ICI minus $I_{n, s-1}$ \\
\hline$P_{\max }(n, s \mid x)$ & prob. sector $n$ ICI of $x$ maximal at stage $s$ & $P_{\text {con }}(n, s \mid x)$ & prob. sector $n$ ICI of $x$ decodable at stage $s$ \\
\hline$P_{\text {sic }}(n, s \mid x)$ & prob. sector $n$ ICI of $x$ cancelled at stage $s$ & $I_{\text {soi }}$ & aggregate ICI from set $\Omega_{\text {soi }}$ \\
\hline$P_{\text {end }, s}$ & prob. SIC process ends at stage $s$ & $I_{\mathrm{end}, s}$ & aggregate ICI from set $\Omega_{\text {soi }}$ at stage $s$ \\
\hline
\end{tabular}

partition, respectively. The shape of sector coverage shown in Fig. 1(b) is given for illustration only. The actual shape is determined by many factors such as antenna directions, antenna patterns and shadowing.

It is noted that the proposed analytical model can work with other variants of FFR schemes, such as soft FFR schemes [4]. Investigation of other FFR schemes with SIC is left for future work.

Classification of users to the center and edge zones is a key aspect of FFR schemes. We use an extended user classification method, which is based on received signal strength (RSS) for downlink communications [38]. In the RSS based method proposed by [38], if the RSS of a user from the user's best from its best serving cell is $3 \mathrm{~dB}$ higher than the received signal strength from the strongest neighbouring cell, the user is classified as cell-center user; otherwise it is classified as cell-edge user. In our user classification the users served by a sector are ranked in term of RSS in decreasing order. Let $N_{\text {ue }}$ denotes the number of users in the sector. The first $\left\lfloor\alpha N_{\mathrm{ue}}\right\rfloor$ users are classified as center users of this sector while the remaining are classified as edge users.

\section{ANALYTICAL MODEL}

\section{A. Overall Analytical Framework}

Our overall analytic framework is presented in Fig. 2. It is general and can takes various cellular deployment geometries, antenna radiation patterns, channels models, user distributions, and link performance models. According to the RSS from the sectors in the network, users are first associated to the sector with the strongest receive signal and classified to either center or edge users. Then we use the analytical model to compute the single sector and aggregate ICIs with FFR in
Section III-B. The aggregate ICI with SIC is modeled in Section IV. Then the aggregate ICI model combined with the given link performance model is used to compute network performance metrics of interests in Section V.

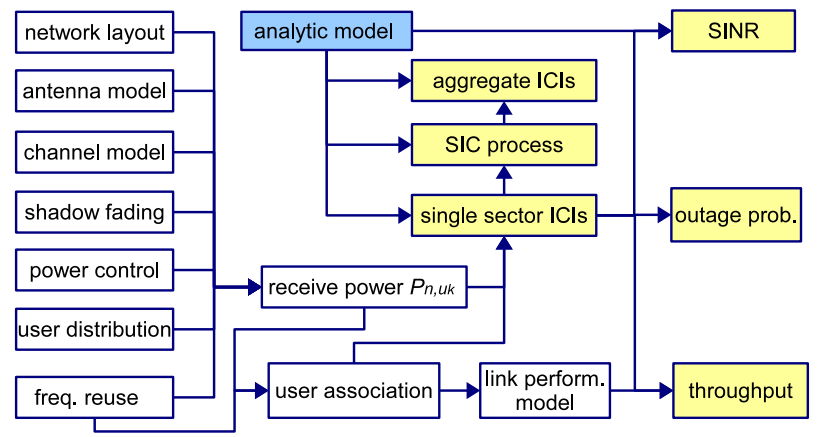

Fig. 2. General analytical framework.

\section{B. Single Sector Interference without SIC}

For the networks with FFR, the uplink ICIs received at the target sites are different for the users at the center and edge zones. For ease of notation we use variable $z$ as subscript to denote the type of zones, with $z=^{\prime} c^{\prime}$ for center zone and $z=^{\prime} e^{\prime}$ for edge zone.

Let $I_{z, n}$ denote the uplink ICI generated from zone type $z$ of sector $n\left(2 \leq n \leq N_{\text {sect }}\right)$ without shadowing and SIC, and its mean and variance denoted by $\bar{I}_{z, n}$ and $\widehat{I}_{z, n}$ respectively, for $z \in\left\{{ }^{\prime} c^{\prime},{ }^{\prime} e^{\prime}\right\}$ and $n=\left[2, N_{\text {sect }}\right]$. The mean $\bar{I}_{z, n}$ and variance $\widehat{I}_{z, n}$ for both zone types of each sector can be computed separately using the approach presented in [23]. In the analytical computation approach, they are obtained by averaging over all the users in zone $z$ of sector $n$ [23]. 
It is noted that the users in the analytical approach are not randomly located and selected as done in the simulation approach. Instead, in [23], the integrations over all possible user locations to compute the mean and variance of $I_{z, n}$ is approximated by a numerical approach. The whole network area is divided into grids of 5 meters by 5 meters. There is one and only one user located at the center of each grid. With the deterministic user locations, the users can be associated to the best serving eNodeBs and be classified to either center or edge users according to the user classification method introduced in Section II.C. After the user association and the user classification, the mean and variance of $I_{z, n}$ can be computed over all the users in zone $z$ of sector $n$. In this way, it will save computation time as there is no need to perform a large number of repeated operations on random user selection and resource allocation used in simulations. Please refer to [23] for more details on the approach of computing the mean and variance of $I_{z, n}$.

It is noted that in the analytical framework presented in Fig. 2, the computation of receive power and user association are not limited to regular cellular layout; in addition, the computation of single sector ICIs without SIC by the approach presented above can handle irregular cellular layout. Therefore the overall analytical framework can model the performance of cellular networks with irregular network layout.

Let $I_{\mathrm{w}, z, n}$ denote the uplink ICI generated from zone type $z$ of sector $n\left(2 \leq n \leq N_{c}\right)$ with shadowing before SIC is applied. We have $I_{\mathrm{w}, z, n}=I_{z, n} \psi_{n}$. A log-normal variable can be uniquely characterised by the mean $\mu$ and standard deviation $\sigma$ for the natural logarithm of the variable. The probability density function $f_{\mathrm{LN}}(x ; \mu, \sigma)$ of the log-normal variable taking value $x$ is expressed by:

$$
f_{\mathrm{LN}}(x ; \mu, \sigma)=\frac{1}{x \sigma \sqrt{2 \pi}} \mathrm{e}^{-\frac{(\ln x-\mu)^{2}}{2 \sigma^{2}}} .
$$

Single sector ICIs without and with shadow fading are assumed to follow log-normal distributions. Let $\mu_{z, n}$ and $\sigma_{z, n}^{2}$ denote the mean and variance of the natural logarithm of $I_{z, n}$, for $n \in\left[1, N_{\text {sect }}\right]$, respectively. $\mu_{z, n}$ and $\sigma_{z, n}^{2}$ can be calculated from $\bar{I}_{z, n}$ and $\widehat{I}_{z, n}$ according to the property of log-normal variables:

$$
\begin{gathered}
\sigma_{z, n}^{2}=\ln \left(1+\frac{\widehat{I}_{z, n}}{\bar{I}_{z, n}^{2}}\right), \\
\mu_{z, n}=\ln \left(\bar{I}_{z, n}\right)-\frac{\sigma_{z, n}^{2}}{2} .
\end{gathered}
$$

Let $\mu_{\mathrm{w}, z, n}$ and $\sigma_{\mathrm{w}, z, n}$ denote the mean and standard deviation of the logarithm of $I_{\mathrm{w}, z, n}$. As single sector ICI with shadow fading is the product of two log-normal variables, we can compute $\mu_{\mathrm{w}, z, n}$ and $\sigma_{\mathrm{w}, z, n}$ for $I_{\mathrm{w}, z, n}$ by:

$$
\begin{aligned}
\mu_{\mathrm{w}, z, n} & =\mu_{z, n}, \\
\sigma_{\mathrm{w}, z, n}^{2} & =\sigma_{z, n}^{2}+\sigma_{\mathrm{w}}^{2} .
\end{aligned}
$$

\section{Aggregate Interference without SIC}

Let $I_{\mathrm{sum}, z}$ denote the aggregate uplink ICI and $\Omega_{\mathrm{ici}, z}$ denote the interfering sectors for zone $z$ users of the target sector, respectively, for $z \in\left\{{ }^{\prime} c^{\prime},{ }^{\prime} e^{\prime}\right\}$. We have:

$$
I_{\mathrm{sum}, z}=\sum_{n \in \Omega_{\mathrm{ici}, z}} I_{\mathrm{w}, z, n} .
$$

For ease of computation $I_{\mathrm{sum}, z}$ is approximated by a lognormal random variable. Let $\mu_{\mathrm{sum}, z}$ and $\sigma_{\mathrm{sum}, z}$ denote the mean and standard deviation of the logarithm of $I_{\mathrm{sum}, z}$, respectively, which can be computed by the Fenton log-normal addition method [39]:

$$
\begin{aligned}
& \sigma_{\mathrm{sum}, z}^{2}=\ln \left[\frac{\sum_{n \in \Omega_{\mathrm{ici}, z}} \mathrm{e}^{2 \mu_{\mathrm{w}, z, n}+\sigma_{\mathrm{w}, z, n}^{2}\left(\mathrm{e}^{\sigma_{\mathrm{w}, z, n}^{2}}-1\right)}}{\left(\sum_{n \in \Omega_{\mathrm{ici}, z}} \mathrm{e}^{\mu_{\mathrm{w}, z, n}+\sigma_{\mathrm{w}, z, n}^{2} / 2}\right)^{2}}\right], \\
& \mu_{\mathrm{sum}, z}=\ln \left(\sum_{n \in \Omega_{\mathrm{ici}, z}} \mathrm{e}^{\mu_{\mathrm{w}, z, n}+\sigma_{\mathrm{w}, z, n}^{2} / 2}\right)-\frac{\sigma_{\mathrm{sum}, z}^{2}}{2} .
\end{aligned}
$$

It is noted that the case of no SIC is a specific case of the general SIC process analyzed in Section IV. The same notations related the aggregate ICI variables are applied in the analysis of the general SIC process in Section IV-D.

\section{Sector and Aggregate Interference With SIC}

After the signals from both desired and interfering users are received, SIC is applied to the aggregate received signal to mitigate interference. In this section the SIC process and the SIC modeling framework are presented first. Then the ICI analysis with SIC is presented.

It is noted that as the SIC process is applied separately and independently to the center and edge zones in parallel, the ICI analysis with SIC is the same for the centre and edge zones except that the set of interfering sectors and the statistics of the sector ICIs are different. Therefore in this section the subscript $z$ (representing the type of centre or edge zone) is dropped for ease of notation and better readability. The analytical approach and the formulae presented in this section are applied later to both centre and edge zones for network performance evaluation in Section V.

\section{A. SIC Process}

In this paper we use an extended abstracted SIC model used in [14] [16], in which the physical layer signal processing details are hidden for model tractability. In the abstracted SIC model used in [14] [16], all the interfering sectors are involved in the SIC process for possible cancellation and a signal decoding condition is checked for SIC success. However, due to the signaling, computation and processing delay constraints, we consider an extended SIC model, in which only a fixed number of sectors of interests (SOI) are the candidates for possible interference cancellation and there is a limit on the maximal number of SIC stages (denoted by $N_{\text {stg }}$ ). In each stage of SIC one and only one signal is picked for possible interference cancellation.

Let $\Omega_{\text {soi }}$ denote the set of SOI, and the size of set $\Omega_{\text {soi }}$ denoted by $N_{\text {soi }}$. Note that the interfering SOI can be chosen as the sectors with the strongest mean ICIs, usually the sectors close to the target sector. The numbers of interfering sectors 
are different for the center and edge sectors, but the sizes of SOI sets for the center and edge sectors can be set to the same. Let $\Omega_{\text {fix }}$ denotes the set of the sectors in the networks that are not to be decoded for cancellation. We have $\Omega_{\text {ici }}=\Omega_{\text {soi }} \cup \Omega_{\text {fix }}$ and $\Omega_{\text {soi }} \cap \Omega_{\text {fix }}=\emptyset$.

For model simplicity the desired signal for the target users from the target sector (sector 1) is not involved in the SIC process for interference cancellation. We believe such treatment does not have a large impact on the model accuracy. In most cases the target signals are decodable without SIC. Therefore the target signals can be subtracted from the aggregated received signal in the first stage of SIC process. It is noted that the proposed model can be extended for the analysis of the SIC process where the target signals are involved in the process.

A flowchart of the actual SIC process used in simulations is presented in Fig. 3(a). The inputs to the SIC process are the ICIs $I_{\mathrm{w}, n}$ from the sectors in sets $\Omega_{\mathrm{soi}}$ and $\Omega_{\mathrm{fix}}$. In the simulations the sector ICI values are generated with random number generators. The subscript $z$ in the $I_{\mathrm{w}, z, n}$ derived in Section III is dropped as discussed previously for the SIC analysis. Firstly the strongest signal from the sectors in set $\Omega_{\text {soi }}$ is decoded. If the signal is not decodable, the SIC process is terminated. Otherwise, the signal is subtracted from the aggregate received signal and the set $\Omega_{\text {soi }}$ is updated by removing the ICI from the decoded sector; and the next stage of SIC is started until the maximal number of SIC stages is reached or the SIC process is terminated.

Mathematically, let $I_{n, s}$ denote the residual interference from sector $n$ after the $s$ th stage SIC, for $s \in\left[0, N_{\text {stg }}\right]$, and $n \in \Omega_{\text {ici. }}$. For $n \in \Omega_{\text {fix }}$, we always have $I_{n, s}=I_{\mathrm{w}, n}$. On the other hand, for $n \in \Omega_{\text {soi }}$, initially for $s=0, I_{n, 0}$ equals to $I_{\mathrm{w}, n}$. For any stage $s>0$, let us introduce intermediate random variables $I_{\mathrm{sic}, n, s}$ with respect to sector $n\left(n \in \Omega_{\mathrm{soi}}\right)$, denoting the sum of the noise and the aggregate residual interference excluding interference $I_{n, s-1}$ for SIC stage $s$, which is computed by:

$$
I_{\text {sic }, n, s}=\sum_{m \in \Omega_{\text {soi }}} I_{m, s-1}+\sum_{m \in \Omega_{\text {fix }}} I_{\mathrm{w}, m}-I_{n, s-1}+\delta^{2} .
$$

It is noted that with the assumption of perfect interference cancellation, the value of $I_{n, s}$ for any $n \in \Omega_{\text {soi }}$ is either $I_{\mathrm{w}, n}$ or 0 . Therefore $I_{\text {sic }, n, s}$ can be expressed by an alternative way:

$$
I_{\text {sic }, n, s}=\sum_{m \in \Omega_{\text {soi }}} I_{\mathrm{w}, m}+\sum_{m \in \Omega_{\mathrm{fix}}} I_{\mathrm{w}, m}-I_{\mathrm{w}, n}+\delta^{2} .
$$

Assume the sector chosen for interference cancellation at SIC stage $s$ is $N_{\max , s}$. Let $\epsilon$ represent the SIC threshold for successful decoding. Then successful cancellation of the chosen interference depends on the condition (16):

$$
I_{N_{\max , s}, s-1} \geq \epsilon I_{\mathrm{sic}, N_{\max , s}, s} .
$$

For $s>0$, the residual ICI $I_{N_{\max , s}, s}$ is updated as follows. If the SIC decoding condition (16) is satisfied, we have $I_{N_{\max , s}, s}=$ 0 ; otherwise $I_{N_{\max , s, s}}=I_{N_{\max , s}, s-1}$. If stage $s$ SIC is successful and $s<N_{\text {stg }}$, the next stage SIC is performed with the following update:

$$
\Omega_{\text {soi }}=\Omega_{\text {soi }} \backslash\left\{N_{\max , s}\right\},
$$

where operator \denotes set minus.

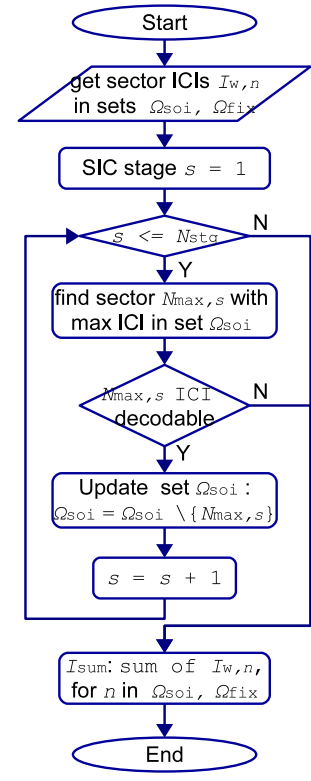

(a) Actual SIC process.

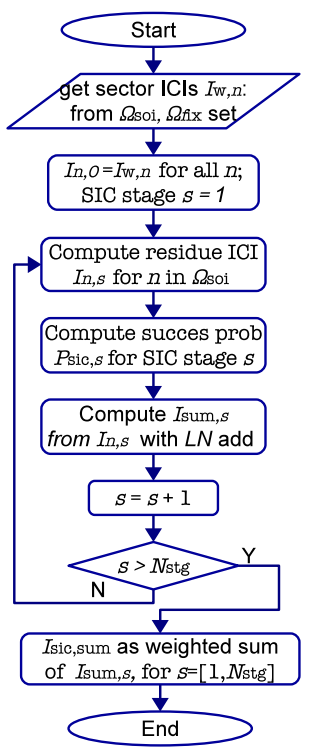

(b) SIC modeling.
Fig. 3. Flowcharts for a) the actual SIC process for simulation; b) SIC modelling.

\section{B. Modeling Framework for SIC Process}

In the actual SIC process there could be a huge number of combinations, if we consider all the possible sectors selected for all the SIC stages, the order of the sectors being processed, and the outcome of the decoding on the ICIs from these selected sectors. These combinations make analytical modeling very difficult. We treat the SIC process alternatively and propose a scalable and low complexity analytical model for it by breaking the close correlation between the SIC stages.

In the actual SIC process used in the simulations a maximal ICI from the SOI set is picked up and cancelled if decodable for each SIC stage, and the sector with cancelled interference is removed from the SOI set. By contrast, in the SIC process analysis, for each SIC stage $s$ the residual sector ICI $I_{n, s-1}$ from each sector $n \in \Omega_{\text {soi }}$ is decoded and cancelled with certain probability, which produces the residual sector ICI $I_{n, s}$. The probability of sector $n$ being cancelled at SIC stage $s$ is denoted by $P_{\text {suc }, n, s}$. A major difference of the modeled SIC process from the actual one is that no sector is removed from set $\Omega_{\text {soi }}$ at the end of any stage SIC processing. We let $P_{\text {suc }, s}$ denote the SIC stage success probability at stage $s$. By this way for each SIC stage (say $s$ ) we need to consider residual sector ICIs left by the previous SIC stage $s-1$ instead of the ICIs prior to stage $s-1$.

A flowchart for the SIC modeling process is presented in Fig. 3(b). In the beginning of each SIC stage $s$ ( $s$ starting from 1 ), for each sector in set $\Omega_{\text {soi }}$, compute the new residual sector ICIs, which is to be presented in Section IV-C. Following the computation of residual sector ICIs the probability $P_{\text {suc }, s}$ of SIC success at stage $s$ is computed, which is to be presented in Section IV-C. Then the aggregate residual ICI for the stage 
(denoted by $I_{\text {sum }, s}$ ) is computed from the residual sector ICIs $I_{n, s}$. The SIC process moves to the next stage. The above process repeats until the maximal number of SIC stages $N_{\text {stg }}$ is reached. Note that there is no update of set $\Omega_{\text {soi }}$ in the loop of Fig. 3(b) as shown in (17) for the actual SIR process. Lastly the aggregate ICI with SIC is computed as the SIC stage success probability weighted sum of the aggregate ICIs at different SIC stages, which is to be presented in Section IV-D.

\section{Residual Sector ICIs}

For the sake of model tractability, we approximate all the residual single sector ICIs $I_{n, s}$ and the aggregate interference $I_{\text {sic }, n, s}$ by log-normal distributions, for $n \in \Omega_{\text {soi }}$ and SIC stages $s \in\left[1, N_{\text {stg }}\right]$. It is noted that despite this and other assumptions of log-normal distributions for the single sector and aggregate ICIs, the analytical model accuracy is preserved as demonstrated by the numerical results presented in Section VI.

Let $\mu_{\text {sic }, n, s}$ and $\sigma_{\text {sic }, n, s}$ denote the mean and standard deviation of the logarithm of the log-normal approximated residual interference $I_{\text {sic }, n, s}$, respectively. There is no closedform expression for the distribution of $I_{\mathrm{sic}, n, s}$ as the sum of single sector ICIs and noise, but it can be reasonably approximated by another log-normal distribution. The values of $\mu_{\text {sic }, n, s}$ and $\sigma_{\text {sic }, n, s}$ can be computed by following formula (13) with the widely used Fenton method [39].

Let $\mu_{n, s}$ and $\sigma_{n, s}$ denote the mean and standard deviation of the logarithm of the log-normal approximated residual interference $I_{n, s}$, respectively. Let $\bar{I}_{n, s}$ and $\widehat{I}_{n, s}$ denote the mean and variation of the residual interference $I_{\mathrm{n}, \mathrm{s}}$, respectively. Next we compute $\bar{I}_{n, s}$ and $\widehat{I}_{n, s}$.

For each SIC stage $s$, let $P_{\text {sic }}(n, s \mid x)$ denote the conditional probability that interference $I_{n, s-1}$ with value $x$ from sector $n$ is cancelled out. Let $P_{\max }(n, s \mid x)$ denote the conditional probability that interference $I_{n, s-1}$ taking value $x$ from sector $n$ is the maximal among the sectors in $\Omega_{\text {soi }}$, and $P_{\text {con }}(n, s \mid x)$ the probability that the interference $I_{n, s-1}$ taking value $x$ meets the SIC condition (16) under the condition of $I_{n, s-1}$ being the maximal among the sectors in $\Omega_{\text {soi }}$. We have

$$
P_{\text {sic }}(n, s \mid x)=P_{\max }(n, s \mid x) P_{\text {con }}(n, s \mid x) .
$$

According to the definition we can compute the probability $P_{\max }(n, s \mid x)$ :

$$
\begin{aligned}
P_{\max }(n, s \mid x) & =\prod_{m \in \Omega_{\text {soi }} \backslash\{n\}} \operatorname{Prob}\left(I_{m, s-1}<x\right) \\
& \cong \prod_{m \in \Omega_{\text {soi }} \backslash\{n\}} \Phi\left(\frac{\ln (x)-\mu_{m, s-1}}{\sigma_{m, s-1}}\right),
\end{aligned}
$$

where $\ln ()$ is the natural logarithm function and $\Phi()$ is the standard normal function. Note that formulae (19) and (20) are derived with the assumption of independent and log-normal distributed interference $I_{m, s-1}$ for $m \in \Omega_{\text {soi }}$.

Computation of the conditional probability $P_{\text {con }}(n, s \mid x)$ is quite challenging. According to definition we can have:

$$
\begin{gathered}
P_{\text {con }}(n, s \mid x)= \\
\operatorname{Prob}\left(\epsilon I_{\text {sic }, n, s}<x \mid x>I_{m, s-1}, m \in \Omega_{\text {soi }} \backslash\{n\}\right)
\end{gathered}
$$

Due to the condition in (21) computing $P_{\text {con }}(n, s \mid x)$ with the above formula directly has a very high computation complexity if not impossible. Next we use a low complexity approximation approach by trying to remove the condition in (21).

Notice that the residual sector ICIs from the SOI set are conditioned to be less than $x$ in the computation of $P_{\text {con }}(n, s \mid x)$ from (21). The condition could be removed by removing a reasonable number of sectors from the SOI set for the computation of $I_{\mathrm{sic}, n, s}$. Intuitively the number of sectors to be removed should be proportional to the inverse of the SIC decoding threshold $\epsilon$. As the conditional residual sector ICIs from the SOI set are less than $x$, the mean of these conditional residual sector ICIs will be much smaller than $x$. Let $N_{\mathrm{v}, n}$ and $\Omega_{\mathrm{v}, n}$ denote the number and the set of SOI sectors being removed with regard to SOI sector $n$. Taking the above concerns into account we set $N_{\mathrm{v}, n}$ heuristically as follows:

$$
N_{\mathrm{v}, n}=\min \left(\left\lfloor\frac{\max \left(\sqrt[3]{-\epsilon_{\mathrm{dB}}}, 0\right)}{\epsilon}\right\rfloor, N_{\text {soi }}-1\right),
$$

where $\epsilon_{\mathrm{dB}}=10 \log 10(\epsilon)$ with interested range of $[0,-10] \mathrm{dB}$. It is noted that the number $N_{\mathrm{v}, n}$ of SOI sectors being removed in the computation of $P_{\text {con }}(n, s \mid x)$ is independent of SIC stage $s$. After the number $N_{\mathrm{v}, n}$ is determined, the set $\Omega_{\mathrm{v}, \mathrm{n}}$ is formed by the $N_{\mathrm{v}, n}$ sectors with the highest interference among the set $\Omega_{\text {soi }}$ but excluding sector $n$.

Let's introduce a new variable $I_{\mathrm{v}, n, s}$, denoting the sum of the noise and the aggregated residual sector ICIs excluding sector $n$ and the removed sectors in set $\Omega_{\mathrm{v}, \mathrm{n}}$, which is defined as:

$$
I_{\mathrm{v}, n, s}=\sum_{m \in \Omega_{\mathrm{soi}} \backslash \Omega_{\mathrm{v}, n}} I_{m, s-1}+\sum_{m \in \Omega_{\mathrm{fix}}} I_{\mathrm{w}, m}-I_{n, s-1}+\delta^{2} .
$$

It is noted that if $N_{\mathrm{v}, n}$ equals to $0, I_{\mathrm{v}, n, s}$ equals to $I_{\text {sic }, n, s}$; if $N_{\mathrm{v}, n}$ is larger than $0, I_{\mathrm{v}, n, s}$ is always smaller than $I_{\mathrm{sic}, n, s}$;

Again the aggregate residual ICIs $I_{\mathrm{v}, n, s}$ are approximated by $\log$-normal distributed random variables, for all $n \in \Omega_{\text {soi }}$ and $s \in\left[1, N_{\mathrm{stg}}\right]$. Let $\mu_{\mathrm{v}, n}$ and $\sigma_{\mathrm{v}, n}$ denote the mean and standard deviation of the logarithm of ICIs $I_{\mathrm{v}, n, s}$, which can be computed with the Fenton log-normal addition method.

Then we compute the conditional probability $P_{\text {con }}(n, s \mid x)$ using the following approximation:

$$
\begin{aligned}
P_{\mathrm{con}}(n, s \mid x) & \approx \operatorname{Prob}\left(\epsilon I_{\mathrm{v}, n, s}<x\right) \\
& \cong \Phi\left(\frac{\ln (x / \epsilon)-\mu_{\mathrm{v}, n, s}}{\sigma_{\mathrm{v}, n, s}}\right) .
\end{aligned}
$$

Note that in (24) $I_{\mathrm{v}, n, s}$ replaces $I_{\mathrm{sic}, n, s}$ in (21) to cancel the impact of the condition present in (21).

It is worth noting that the operation of removing $N_{v, n}$ sectors from the set $\Omega_{\text {soi }}$ is purely for the purpose of simplifying the computation of the conditional probability $P_{\text {con }}(n, s \mid x)$. With this treatment, the original formula (21) used to compute $P_{\text {con }}(n, s \mid x)$ is replaced by (25), which makes the analytic model more tractable without large impact on analytic model accuracy. However, the overall SIC process and the computation of the aggregate ICI are not affected by the treatment. In 
the formula (28) the aggregate ICI with SIC is computed over all the sectors in the set $\Omega_{\text {soi }}$ with no sector being removed.

Now the mean $\bar{I}_{n, s}$ of residual single sector interference $I_{n, s}$ can be computed by (26):

$$
\bar{I}_{n, s}=\int_{0}^{\infty} x\left[1-P_{\text {sic }}(n, s \mid x)\right] f_{\mathrm{LN}}\left(x ; \mu_{n, s-1}, \sigma_{n, s-1}\right) \mathrm{d} x,
$$

and the variance $\widehat{I}_{n, s}$ is computed by :

$$
\begin{aligned}
\widehat{I}_{n, s}= & \int_{0}^{\infty}\left\{\left(x-\bar{I}_{n, s}\right)^{2}\left[1-P_{\text {sic }}(n, s \mid x)\right]\right. \\
& \left.+\bar{I}_{n, s}^{2} P_{\text {sic }}(n, s \mid x)\right\} f_{\mathrm{LN}}\left(x ; \mu_{n, s-1}, \sigma_{n, s-1}\right) \mathrm{d} x .
\end{aligned}
$$

It is noted that for the case of $s=1$, we have $\mu_{n, s-1}=\mu_{z, n}$ and $\sigma_{n, s-1}=\sigma_{z, n}$.

From the mean $\bar{I}_{n, s}$ and the variance $\widehat{I}_{n, s}, \mu_{n, s}$ and $\sigma_{n, s}$ can be computed by following formulae (8) and (9).

\section{Aggregate ICI with SIC}

Now that the log-normal distribution variables $\left(\mu_{n, s}\right.$ and $\sigma_{n, s}$ ) for the residual sector ICIs $I_{n, s}$ are computed, we can compute the aggregate residual ICIs from sectors in the set $\Omega_{\text {soi }}$ for stage $s \in\left[0, N_{\mathrm{stg}}\right]$, which is denoted by $I_{\mathrm{soi}, s} . I_{\mathrm{soi}, s}$ is the sum of multiple approximated log-normal variables and computed by:

$$
I_{\mathrm{soi}, s}=\sum_{n \in \Omega_{\mathrm{soi}}} I_{n, s} .
$$

Let $\bar{I}_{\mathrm{soi}, s}$ and $\widehat{I}_{\mathrm{soi}, s}$ denote the mean and variation of $I_{\mathrm{soi}, s}$, which can be computed according to (28).

Next we compute the probability $P_{\text {suc }, s}$ that the SIC process at a general stage $s\left(s \in\left[1, N_{\mathrm{stg}}\right]\right)$ is successful, which means there is one and exactly one sector from the set $\Omega_{\text {soi }}$ is successfully decoded and cancelled. Let $P_{\text {suc }, n, s}$ denote the probability that interference $I_{n, s-1}$ is the maximal among the set $\Omega_{\text {soi }}$ and is cancelled out at SIC stage $s$, for $n \in \Omega_{\text {soi }}$ and $s \in\left[1, N_{\text {stg }}\right]$. The success probability $P_{\text {suc }, n, s}$ is computed by:

$$
P_{\text {suc }, n, s}=\int_{0}^{\infty} P_{\text {sic }}(n, s \mid x) f_{\mathrm{LN}}\left(x ; \mu_{n, s}, \sigma_{n, s}\right) \mathrm{d} x,
$$
by:

Then the SIC stage success probability $P_{\mathrm{suc}, s}$ is computed

$$
P_{\mathrm{suc}, s}=\sum_{n \in \Omega_{\mathrm{soi}}} P_{\mathrm{suc}, n, s} .
$$

Let $P_{\text {end, } s}$ denote the probability that the SIC process ends at exactly at stage $s$, for $s \in\left[1, N_{\text {stg }}\right]$, which can be computed by:

$$
P_{\text {end }, s}= \begin{cases}1-P_{\mathrm{suc}, 1}, & s=1 ; \\ \left(1-P_{\mathrm{suc}, s+1}\right) \prod_{l=1}^{s} P_{\mathrm{suc}, l}, & s=\left[2, N_{\mathrm{stg}}-1\right] ; \\ \prod_{l=1}^{N_{\mathrm{stg}}-1} P_{\mathrm{suc}, l}, & s=N_{\mathrm{stg}} .\end{cases}
$$

Let $I_{\text {soi }}$ denote the aggregate ICI from the set $\Omega_{\text {soi }}$ at the end of the SIC process, which is the sum of the aggregate residual
ICIs $I_{\mathrm{soi}, s}$ weighted by the probability $P_{\mathrm{end}, s}$ and computed by:

$$
I_{\mathrm{soi}}=\sum_{s=1}^{N_{\mathrm{stg}}} P_{\mathrm{end}, s} I_{\mathrm{soi}, s}
$$

Let $\bar{I}_{\text {soi }}$ and $\widehat{I}_{\text {soi }}$ denote the mean and variation of $I_{\text {soi }}$, which can be computed by:

$$
\begin{aligned}
& \bar{I}_{\mathrm{soi}}=\sum_{s=1}^{N_{\mathrm{stg}}} P_{\mathrm{end}, s} \bar{I}_{\mathrm{soi}, s}, \\
& \widehat{I}_{\mathrm{soi}}=\sum_{s=1}^{N_{\mathrm{stg}}} P_{\mathrm{end}, s}\left(\widehat{I}_{\mathrm{soi}, s}+\bar{I}_{\mathrm{soi}, s}^{2}\right)-\bar{I}_{\mathrm{soi}}^{2} .
\end{aligned}
$$

Again we approximate the aggregate ICI $I_{\text {soi }}$ by a lognormal random variable. Let $\mu_{\text {soi }}$ and $\sigma_{\text {soi }}$ denoting the mean and standard deviation of the logarithm of $I_{\text {soi }}$, respectively, which can be computed by the Fenton log-normal addition method.

Finally we can compute the aggregate ICI $I_{\text {sum }}$ from all the interfering sectors with SIC process.

$$
I_{\text {sum }}=I_{\text {soi }}+\sum_{n \in \Omega_{\mathrm{fix}}} I_{n, s} .
$$

$I_{\text {sum }}$ is approximated by a log-normal random variable. Then the mean $\mu_{\text {sum }}$ and standard deviation $\sigma_{\text {sum }}$ of the logarithm of $I_{\text {sum }}$ can be computed using the Fenton log-normal addition method.

\section{Network Performance}

The active users located in the same FFR zones (center or edge zone) of the target sector (sector 1) are expected to experience aggregate ICIs with the same statistics, which have been assumed to follow log-normal distribution. Let $I_{\text {sum }, z}$ denote the aggregate uplink ICI of the target sector for the zone of type $z\left(z \in\left\{{ }^{6} c^{6},{ }^{6} e^{6}\right\}\right)$. Let $\mu_{\text {sum }, z}$ and $\sigma_{\text {sum }, z}$ denote the mean and standard variation of $I_{\text {sum }, z}$, which can be computed using the approach presented in Section IV.

Consider a general user $u$ in zone $z$ of the target sector. The SINR $\gamma_{z, u}$ for user $u$ is calculated as:

$$
\gamma_{z, u}=\frac{\mathcal{P}_{1, u}}{I_{\mathrm{sum}, z}+\delta^{2}} .
$$

Let $I_{z}=I_{\text {sum }, z}+\delta^{2}$. Treating $\delta^{2}$ as a log-normal variable with mean of $\log \left(\delta^{2}\right)$ and standard deviation of 0 for its logarithm, we can approximate $I_{z}$ by a new log-normal variable, with mean and standard deviation denoted by $\mu_{z}$ and $\sigma_{z}$ for its logarithm, respectively. $\mu_{z}$ and $\sigma_{z}$ can be calculated by Fenton log-normal addition method [39].

Now as $\mathcal{P}_{1, u}$ and $I_{z}$ are two independent log-normal variables, the SINR $\gamma_{z, u}$ of user $u$ in zone $z$ is also a log-normal variable. Let $\mu_{z, u}$ and $\sigma_{z, u}$ denote the mean and standard deviation of the logarithm of $\gamma_{z, u}$, which can be computed by:

$$
\begin{aligned}
\mu_{z, u} & =\ln \left(P_{1, u}^{\mathrm{r}}\right)-\mu_{z}, \\
\sigma_{z, u}^{2} & =\sigma_{\mathrm{w}}^{2}+\sigma_{z}^{2} .
\end{aligned}
$$


Let $\bar{\gamma}_{z, u}$ and $\widehat{\gamma}_{z, u}$ denote the mean and variance of SINR of user $u$ in zone $z$, respectively, which can be computed using formulae:

$$
\begin{aligned}
& \bar{\gamma}_{z, u}=e^{\mu_{z, u}+\sigma_{z, u}^{2} / 2}, \\
& \widehat{\gamma}_{z, u}=\left(e^{\sigma_{z, u}^{2}}-1\right) \bar{\gamma}_{z, u} .
\end{aligned}
$$

Let $(r, \theta)$ denote the polar coordinates of general user $u$ from zone $z$ of the target sector. The mean SINR for users in zone $z$ (denoted by $\gamma_{\text {net }, z}$ ) can be obtained by integrating the single user SINR $\gamma_{z, u}$ over zone $z$ of sector 1 :

$$
\gamma_{\text {net }, z}=\iint_{\mathcal{A}_{1}^{z}} \gamma_{z, u} r \mathrm{~d} r \mathrm{~d} \theta .
$$

It is noted that $\gamma_{z, u}$ is a function of coordinates $(r, \theta)$ of user $u$ in zone $z$.

For any instantaneous SINR, we suppose that there is a link layer performance model $\mathcal{F}($.$) that maps a SINR to spectral$ efficiency (bps/Hz). Let $\mathcal{C}_{z, u}$ and $\overline{\mathcal{C}}_{z, u}$ denote the instantaneous and average spectrum efficiency (bps/Hz) of user $u$ in zone $z$, respectively, which can be calculated with consideration of users in both center and edge zones:

$$
\begin{aligned}
\mathcal{C}_{z, u} & =\mathcal{F}\left(\gamma_{z, u}\right), \\
\overline{\mathcal{C}}_{z, u} & =\int_{0}^{\infty} \mathcal{F}(x) f_{\mathrm{LN}}\left(x ; \mu_{z, u}, \sigma_{z, u}\right) \mathrm{d} x .
\end{aligned}
$$

Let $\mathcal{C}_{\text {sect }}$ denote the average spectrum efficiency $(\mathrm{bps} / \mathrm{Hz})$ of sector $\mathcal{A}_{1}$ which is calculated by:

$$
\begin{gathered}
\mathcal{C}_{\text {sect }}= \\
\frac{\iint_{\mathcal{A}_{1}^{C}} r \mathrm{~d} r \mathrm{~d} \theta \iint_{\mathcal{A}_{1}^{C}} \overline{\mathcal{C}}_{c, u} r \mathrm{~d} r \mathrm{~d} \theta+\iint_{\mathcal{A}_{1}^{e}} r \mathrm{~d} r \mathrm{~d} \theta \iint_{\mathcal{A}_{1}^{e}} r \overline{\mathcal{C}}_{e, u} / 3 \mathrm{~d} r \mathrm{~d} \theta}{\iint_{\mathcal{A}_{1}} r \mathrm{~d} r \mathrm{~d} \theta} .
\end{gathered}
$$

Note that the denominator 3 in the above formula reflects the impact of the frequency reuse factor of 3 for edge zones. The mean site throughput (denoted by $\eta_{\text {site }}$ ) in bps can be computed with network bandwidth $W$ by:

$$
\eta_{\text {site }}=3 \mathcal{C}_{\text {sect }} W
$$

Suppose that a user is out of service if its instantaneous SINR is lower than a given outage threshold $\gamma_{\text {out }}$. Let $\mathcal{O}_{z, u}$ denote the outage probability of a general user $u$ in zone $z$ of sector 1 , which can be calculated by:

$$
\mathcal{O}_{z, u}=\int_{0}^{\gamma_{\text {out }}} f_{L N}\left(x ; \mu_{z, u}, \sigma_{z, u}\right) d x
$$

Then the network wide mean outage probability (denoted by $\mathcal{O}_{\text {net }}$ ) can be calculated by:

$$
\mathcal{O}_{\text {net }}=\frac{\iint_{\mathcal{A}_{1}^{c}} r \mathrm{~d} r \mathrm{~d} \theta \iint_{\mathcal{A}_{1}^{c}} \overline{\mathcal{O}}_{c, u} r \mathrm{~d} r \mathrm{~d} \theta+\iint_{\mathcal{A}_{1}^{e}} r \mathrm{~d} r \mathrm{~d} \theta \iint_{\mathcal{A}_{1}^{e}} \overline{\mathcal{O}}_{e, u} r \mathrm{~d} r \mathrm{~d} \theta}{\iint_{\mathcal{A}_{1}} r \mathrm{~d} r \mathrm{~d} \theta} .
$$

It is noted that similar to the computation of the mean and variance of sector ICI, the numerical technique is applied to compute the network performance instead of applying formulae (44) and (47) directly. In this case, the target sector (sector 1) area is divided into grids of 5 meters by 5 meters.
There is one and only user located at each grid in sector 1 . These users are classified to either center user or edge user according to the classification method introduced in Section II.C. Then the spectrum efficienc and outage probability for sector 1 can be computed by averaging over all the users in sector 1 .

\section{NUMERIC RESULTS}

In this section the performance of the LTE uplink communications with and without FFR and SIC are evaluated, and the impact of various FFR and SIC related parameters is assessed.

\section{A. System configurations}

Network performance is evaluated and compared using the proposed analytical model as well as simulations. Analytical results are obtained using Matlab. Simulation results are averaged over $10^{4}$ simulations runs. Table II presents the most relevant system parameters.

In the simulations, the users are uniformly distributed within the coverage area of the sectors. Moreover, we want to highlight that while we assumed a regular hexagonal site placement and a uniform distribution of users in this paper, the proposed analytic model can be extended to work with other site placements and user distributions.

For the numerical evaluation, we use a spectral efficiency function $\mathcal{F}(x)$, which approximates an abstracted LTE link level model developed from the LTE link level Simulator [20], with $2 \times 2$ antenna mode, open loop spatial multiplexing (OLSM) and adaptive modulation and coding (AMC). The original LTE link model (presented in Fig.9 of [20]) mapping channel SNR (dB) to spectral efficiency $(\mathrm{bps} / \mathrm{Hz})$ is approximated by a polynomial function presented in [8]. It is noted that the analytical models show good prediction accuracy with other abstracted link level models as well, but the results are not presented here due to limited space.

The network performance metrics of interests include mean site throughput, the 5th tile site throughput and mean outage probability. It is worth noting that the 5th tile site throughput is a new performance metric introduced in this paper. Traditionally the 5th tile user throughput has been widely used to measure throughput of users located at the edge of wireless networks, usually used as an indication of user fairness. The 5 th tile user throughput is defined as the minimal throughput of the users below which exactly $5 \%$ of the users's throughput are found. The following simple algorithm can be used to determine the 5th tile user throughput:

- Order the user throughputs in increasing order.

- Discard the lowest 5\% throughputs.

- The next lowest throughput is the 5\% tile user throughput

However, the 5th tile user throughput is highly dependent on the number of users in the networks, which makes it not a good candidate for fair performance comparison. Take one simple example. Suppose the network throughput is $10 \mathrm{Mbps}$, equally shared by 10 users with a 5th tile user throughput of 1 Mbps. If there are 20 users, the 5th tile user throughput changes to $0.5 \mathrm{Mbps}$, which is different from that with 10 users. The problem is even worse with FFR where the number of users in 
TABLE II

SYSTEM SETTINGS

\begin{tabular}{|c|c|c|c|}
\hline Parameters & Value & Parameters & Value \\
\hline Carrier freq. & $2000 \mathrm{MHz}$ & Bandwidth $W$ & $5 \mathrm{MHz}$ \\
\hline Number of sites & 19 & Inter-site distance $R_{\mathrm{ISD}}$ & $500 \mathrm{~m}$ \\
\hline Max transmit power $P_{\max }$ & $250 \mathrm{~mW}$ & Target receive power $P_{\text {target }}$ & $-50 \mathrm{dBm}$ \\
\hline Antenna height & $25 \mathrm{~m}$ & User height & $1.5 \mathrm{~m}$ \\
\hline Max antenna gain & $15.5 \mathrm{dBi}$ & Antenna front to back ratio & $25 \mathrm{~dB}$ \\
\hline Horizontal HPBW $\theta_{3 d B}$ & $65^{\circ}$ & Vertical HPBW $\vartheta_{3 d B}$ & $11.5^{\circ}$ \\
\hline Antenna downtilt $\theta_{\text {down }}$ & $10.38^{\circ}$ & Shadowing std dev & $6 \mathrm{~dB}$ \\
\hline FFR bandwidth partition $\alpha$ & 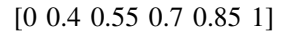 & Noise power $\delta^{2}$ & $-116 \mathrm{dBm}$ per $\mathrm{PRB}$ \\
\hline Power compensation factor $\beta$ & 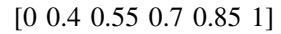 & SIC max number of stages $N_{\mathrm{stg}}$ & {$\left[\begin{array}{llll}0 & 1 & 2 & 3\end{array}\right]$} \\
\hline SIC SOI number $N_{\text {soi }}$ & {$\left[\begin{array}{lll}5 & 10 & 15\end{array}\right]$} & SIC decode threshold $\epsilon$ & {$\left[\begin{array}{llll}0 & -2 & -4 & -8\end{array}\right] \mathrm{dB}$} \\
\hline
\end{tabular}

the edge zone can be varied with different bandwidth partition coefficient $\alpha$.

Therefore we propose the so-called the 5th tile site throughput performance metric. Firstly we compute the 5 th tile user throughput in one sector by applying the algorithm introduced previously on both cell center and cell edge users. Then we compute the 5th tile site throughput as the product of the 5th tile user throughput, the number of users in the sector and the number of sectors per site. In this way we can have a comparable 5th tile performance metric, which is not affected by the number of users in the networks.

\section{B. Network Performances with FFR and Power Compensation}

1) Impact of FFR: Firstly we investigate the impact of FFR on network performance. The FFR bandwidth partition parameter $\alpha$ is varied from 0 to 1 . It is noted that $\alpha=1$ and $\alpha=0$ correspond to the integer frequency reuse with reuse factor of 1 and 3, respectively.

Fig. 4 show the network performance against the FFR parameter $\alpha$, with other typical settings of power compensation factor $\beta=0.4$, SIC decoding threshold $\epsilon=-4 \mathrm{~dB}$, and number of SOI $N_{\text {soi }}=15$. The outage SINR threshold is $-4 \mathrm{~dB}$. The maximal number of SIC stages $N_{\text {stg }}$ is set to 0,1,2 and 3 . Note that the setting $N_{\mathrm{stg}}=0$ corresponds to the case of SIC not applied.

From these results shown in Fig. 4 we have the follow observations.

a) The analytical results closely match simulation results, with an average absolute model deviation of $0.97 \%$ for the mean site throughput. This fact shows the high accuracy of our proposed analytical model. Apart from the easy reproducibility, the analytical approach is significantly fast than simulation approach. Simulations took more than 2 days to produce the simulation results presented in this paper, while analytical models took less than 5 minutes. Therefore this analytical model can be used as an effective tool to predict network performance in a fast and reliable manner. For example, it can be used to find proper settings of parameters for the sector antennas, FFR bandwidth partition, uplink power control and SIC by quickly searching over different candidate configurations.

$b$ ) In the case of SIC not applied $\left(N_{\mathrm{stg}}=0\right)$, the highest throughput comes at bandwidth partition coefficient $\alpha=0.7$ according to Fig. 4(a), and mean outage probability monotonically increases to 0.25 with increasing $\alpha$. As there is not much difference on the throughput for $\alpha$ taking values in [0.4, 0.7], a relative low value of $\alpha$ (such as 0.4) is preferred for FFR without use of SIC. However with the use of SIC, smaller $\alpha$ is no longer favored. For all the settings of $N_{\mathrm{stg}}>0$, the throughput is stable for $\alpha=[0.55,1]$ but obviously higher than that with $\alpha=0.4$. On the other hand, although the outage probability increases with $\alpha$, the largest outage probability obtained at $\alpha=1$ is $0.15,0.11$ and 0.105 for $N_{\text {stg }}=1,2$ and 3, respectively, which can be tolerable and much lower compared to the one of 0.25 for $N_{\text {stg }}=0$. And the 5th tile site throughput shown in Fig. 4(b) is much higher with $\alpha=1$. Therefore it may be concluded that SIC has dominant influence over FFR. If SIC is applied a higher $\alpha$ or even a universal frequency reuse should be used.

c) Integer frequency reuse with reuse factor of 3 (i.e, $\alpha=0$ ) always gives the lowest outage probability compared to other $\alpha$ settings, and has the second highest 5th tile network throughput, which come from much weaker intercell interference due to larger frequency reuse distance. But there is a very high cost on the frequency utilization efficiency: the mean site throughput with $\alpha=0$ is more than $30 \%$ lower than that with $\alpha=1$. Therefore $\alpha=0$ should not be used no matter whether SIC is applied or not.

2) Impact of Uplink Power Compensation: Next we investigate the impact of uplink power compensation on network performance. This time the power compensation factor $\beta$ is varied from 0 to 1 . Fig. 5 shows the network performance against power compensation factor $\beta$ with FFR parameter $\alpha=0.55$. The SIC parameters remains unchanged as set in Section VI-B1. Compared to the results shown in Fig. 4, the results against $\beta$ gives a relative easy choice on $\beta$ value. For example with $N_{\text {stg }}=0$, the site throughput shown in Fig. 5(a) increases significantly from 8 Mbps with $\beta=0$ to $13.8 \mathrm{Mbps}$ with $\beta=0.4$, then it decreases slowly with increasing $\beta$. There are similar trends for the other $N_{\text {stg }}$ settings. The 5th tile site throughput shown in Fig. 5(b) changes with $\beta$ in a similar way as site throughput, but the maximal 5th tile site throughput is obtained at $\beta=0.85$. On the other hand, the outage probability shown in Fig. 5(c) decreases significantly from 0.35 with $\beta=0$ to 0.1 with $\beta=0.4$ for $N_{\text {stg }}=0$, and then stabilizes for $\beta$ in [0.4, $1]$.

It can be concluded that power compensation does show large impact on the uplink communications of LTE network, but it is relatively easy to pick $\beta=0.4$ as the best setting, which will be used in the following investigation on the impact of 
SIC parameters.

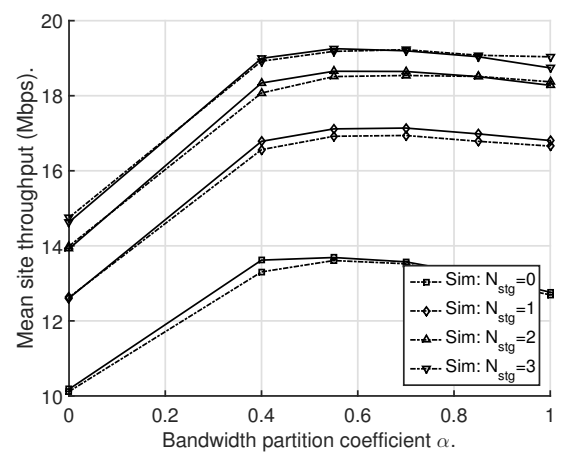

(a) Mean site throughput.

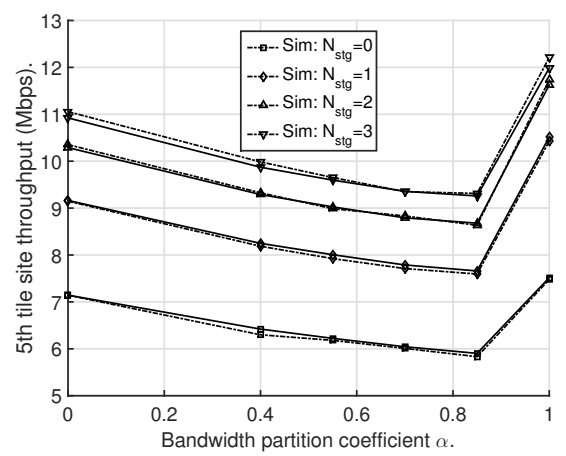

(b) The 5th tile site throughput.

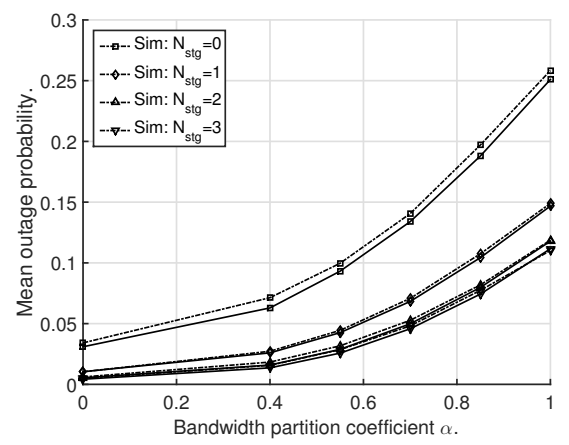

(c) Outage probability.

Fig. 4. Network performance against FFR bandwidth partition coefficient $\alpha$, with uplink power compensation factor $\beta=0.4$. a) mean site throughput; b) the 5 th tile site throughput; c) mean outage probability. Dashed line: simulation ('Sim'); Solid line: model.

\section{Network Performance with SIC}

In this subsection we study the impact of SIC parameters (the maximal number of SIC stages $N_{\text {stg }}$, the number of SOI $N_{\text {soi }}$ and the SIC decoding threshold $\epsilon$ ) on network performance.

1) Impact of Maximal Number of Stages $N_{\text {stg }}$ : In Fig. 4 and Fig. 5, the results with different settings of $N_{\text {stg }}$ versus the FFR parameter $\alpha$ and power compensation factor $\beta$ have been presented, respectively. It can be observed from Fig. 4 that across the range $[0,1]$ of $\alpha$, there is consistent significant improvement with SIC on network performance (mean throughput, the 5th tile throughput and outage probability) over these without SIC. The throughput gain of SIC with $N_{\mathrm{stg}}=1$ over no SIC is more than $25 \%$ at $\alpha=0.55$. With increasing $N_{\text {stg }}$

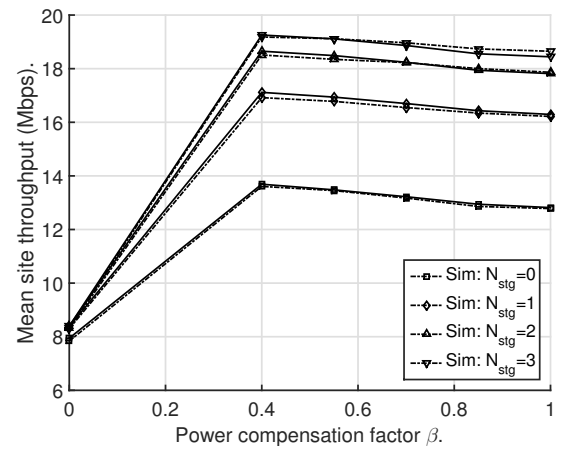

(a) Mean site throughput.

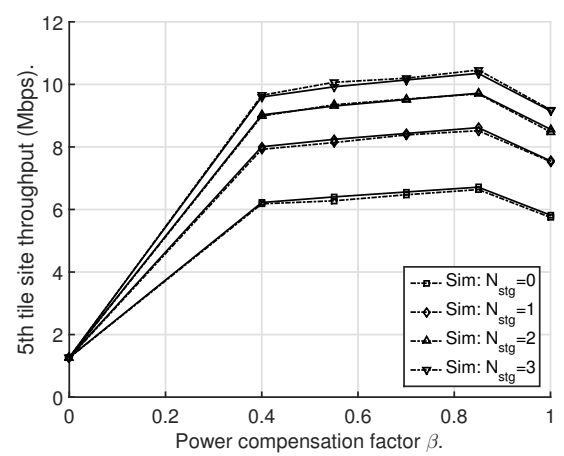

(b) The 5th tile site throughput.

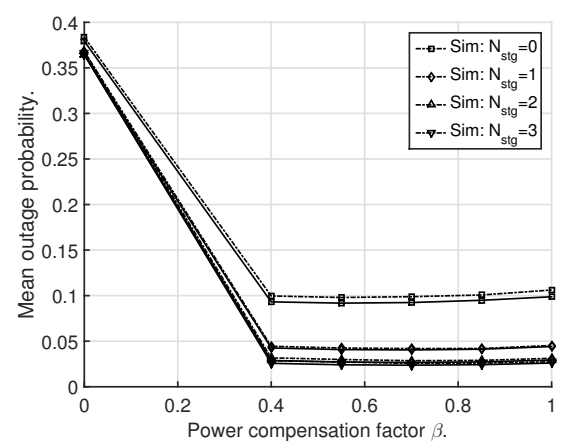

(c) Outage probability.

Fig. 5. Network performance against uplink power compensation $\beta$, with FFR bandwidth partition coefficient $\alpha=0.4$. a) mean site throughput; b) the 5 th tile site throughput; c) mean outage probability. Dashed line: simulation ('Sim'); Solid line: model.

there are further but decreasing throughput gains. The further throughput gain of SIC with $N_{\text {stg }}=2$ and $N_{\text {stg }}=3$ over no SIC is about $12 \%$ and $6 \%$, respectively. The further performance gain with $N_{\text {stg }}$ larger than 3 is diminishing, which are not presented in this paper. Similar trends are observed for the 5th tile throughput. For the outage probability performance, there is a big drop from no SIC $\left(N_{\text {stg }}=0\right)$ to SIC with $N_{\text {stg }}=1$, but only slight further reduction with $N_{\text {stg }}$ larger than 1 . Very similar impact of $N_{\text {stg }}$ on network performance is observed for the results presented in Fig. 5.

The above observations show that the major performance gains of SIC can be achieved with relatively small $N_{\text {stg }}$ (3 or less), which is strongly favored as each stage of SIC process takes considerable time and using small $N_{\text {stg }}$ can avoid excessive processing delay but get the most of SIC benefits. 
2) Impact of Number of SOI $N_{\text {soi }}$ : A large number of SOI for SIC process can have a positive impact on network performance improvement but a negative impact with increased signalling overhead and SIC processing delay. In the previous presented results, the number of SOI $N_{\text {soi }}$ is set to 15 . In this subsection further results are presented with $N_{\text {soi }}$ set to 5, 10 and 15, to study the impact of $N_{\text {soi }}$ settings. Fig. 6 presents network performance versus SIC stage number $N_{s t g}$ with FFR parameter $\alpha$ set to 0.55 . Power compensation factor $\beta$ is 0.4 and the SIC decoding threshold $\epsilon$ is $-4 \mathrm{~dB}$. In the figures analytical results (in white bars) are presented next to the simulation results (in colorful bars for different $N_{\text {soi }}$ ). As $N_{\text {stg }}=0$ corresponds to the case of SIC being not applied, the number of SOI is not applicable to the case of setting $N_{\mathrm{stg}}=0$.

It can be observed again that analytical results have good agreement with simulation results. The results presented in Fig. 6 confirm the effectiveness of SIC and the clear impact of SIC stage number $N_{\text {stg. }}$. Compared to the SIC stage number $N_{\text {stg }}$, the number of SOI has a much weaker impact and the impact depends highly on the stage number $N_{s t g}$. For the case of $N_{\text {stg }}=1$, the network performance differences with different $N_{\text {soi }}$ is almost negligible. For the case of $N_{s t g}$ being 2 and 3, there is around $1 \mathrm{Mbps}$ improvement on both site throughput and the 5th tile throughput with $N_{\text {soi }}=10$ over that with $N_{\text {soi }}=5$, but very little further improvement with $N_{\text {soi }}=15$.

The results suggest that it is not necessary to have a large number of sectors selected for interference cancellation. Using 10 or less closest interfering sectors for SIC can get the most of SIC benefits, which helps control the signalling overheads and SIC processing delay.

3) Impact of SIC Decoding Threshold: SIC decoding threshold $\epsilon$ has a great influence on the successful interference cancellation and network performance. Unlike the SIC parameters $N_{s t g}$ and $N_{s o i}$, which are relatively easy to configure and optimize during system operations, SIC decoding threshold $\epsilon$ is closely related to hardware/software capabilities and hard to configure.

Table III presents the mean site throughput versus SIC decoding threshold $\epsilon$ of $0,-2,-4$ and $-8 \mathrm{~dB}$. Simulation results on the mean throughput are presented in the columns with label 'Sim.'. Analytical results are presented in the columns with label 'Mod.', respectively. The columns with label 'Gain' following the column 'Sim.' show the gain of throughput in percents with a given number of stage $N_{\text {stg }}$ over that with $N_{\text {stg }}=0$. The columns with label 'Dev.' show the deviation of analytical results to simulation results in percents. The data is organized by four blocks of columns corresponding to the $N_{\text {stg }}$ settings $(0,1,2$ and 3$)$ and four blocks of rows corresponding to the $\epsilon$ settings. For each block of rows, there are four combinational settings on the FFR parameter $\alpha(0.55$ and 1.0) and power compensation factor $\beta$ (0.4 and 1). The number of SOI $N_{\text {soi }}$ is set to 15 . It is noted that the block of results with $N_{\mathrm{stg}}=0$ are the same for different decoding threshold $\epsilon$, as decoding threshold $\epsilon$ has no impact when SIC is not applied $\left(N_{\text {stg }}=0\right)$.

According to the results presented in Table III, the analytical model has a high accuracy on predicting the network performances for various configurations of system and SIC param-

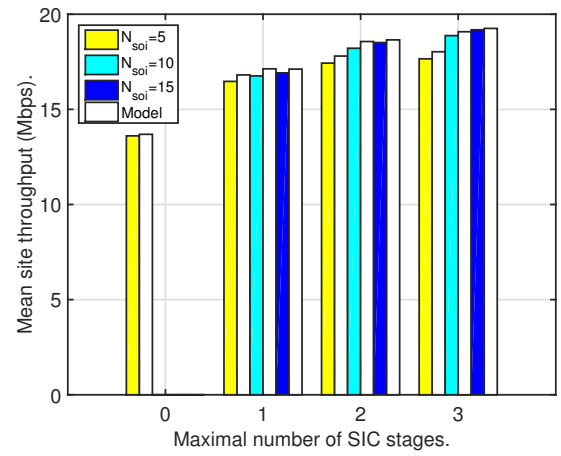

(a) Mean site throughput.

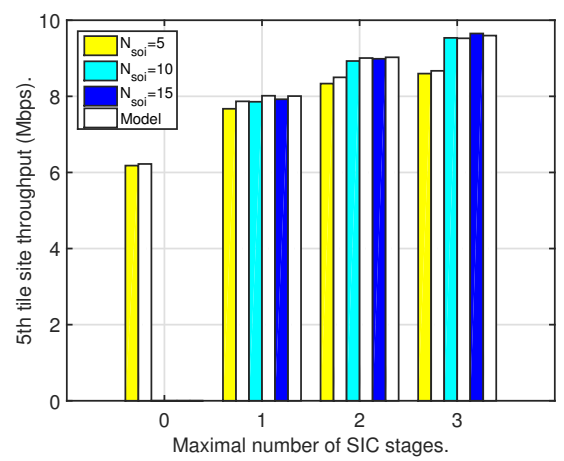

(b) The 5th tile site throughput.

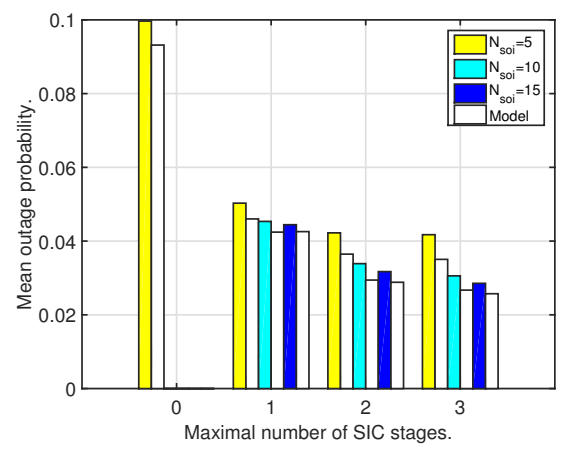

(c) Outage probability.

Fig. 6. Site throughput versus maximal number of SIC stages, with uplink power compensation factor a) $\alpha=0.55$; b) $\beta=0.4$. Simulation (color bar) and analytical (white bar) results are presented side by side for $N_{\text {soi }}=5,10,15$.

eters. And the results show that the SIC decoding threshold $\epsilon$ has a significant impact on the network performance gain of SIC.

As found out in Section VI-B1 and Section VI-B2, an overall good setting for FFR bandwidth partition coefficient $\alpha$ is 0.55 and a good setting for power compensation factor $\beta$ is 0.4 . Next we will focus on the study of the impact of decoding threshold with the configurations of $\alpha=0.55$ and $\beta=0.4$. With $0 \mathrm{~dB}$ decoding threshold and given configurations of $\alpha$ and $\beta$, the gain of the mean site throughput over $N_{\mathrm{stg}}$ in percents is $14.52,15.62$ and 16.25 , for $N_{\mathrm{stg}}=1,2$ and 3, respectively. With $-2 \mathrm{~dB}$ decoding threshold, the throughput gain in percents becomes 19.81, 25.2 and 26.23, for $N_{\text {stg }}=1$, 2 and 3, respectively. With $-4 \mathrm{~dB}$ decoding threshold, the throughput gain in percents has a large improvement to 24.34 , 36.03 and 40.96 , for $N_{\text {stg }}=1,2$ and 3, respectively. With $-8 \mathrm{~dB}$ 
decoding threshold, the throughput gain in percents becomes $28.35,47.65$ and 63.02 , for $N_{\mathrm{stg}}=1,2$ and 3, respectively. It is observed that the lower decoding threshold, the much higher performance gains with large $N_{\text {stg }}$.

\section{CONCLUSION}

Successive interference cancellation (SIC) and fractional frequency reuse (FFR) are two representative inter-cell interference mitigation techniques. In this paper we studied the joint application of FFR and SIC to LTE uplink communications, and develop a general analytical model to investigate their interactions and impact on network performance. In the model inter-cell interference (ICI) from the individual cell sectors were approximated by log-normal random variables, which enables low complexity computation and model tractability but preserves the accuracy on modeling the aggregate ICI with FFR and SIC. Then network performance in terms of SINR, site throughput and outage probability was computed. The key dynamic functionalities and variables, including FFR bandwidth partition, uplink power control, sector antennas and SIC related parameters, were taken into account in the model. The model was fast and validated by simulations, with less than $1 \%$ modelling deviation. From the numerical results it was observed that both FFR and SIC could largely improve network performance, and SIC had a dominant impact. In addition it was found that the SIC parameters had significant impact on network performance, and the most of SIC gains could be obtained with relatively small number of stages of SIC on the ICIs from ten or less sectors, which is favored for practical applications with signalling and processing delay constraints.

\section{ACKNOWLEDGMENT}

The authors would like to thank the reviewers for their constructive comments. The work of J. He and Z. Tang was supported by FP7 Grant DETERMINE (FP7-PEOPLE2012-IRSES under grant number 318906) and EU Horizon2020 project COSAFE (H2020-MSCA-RISE-2018 under grant number 824019). The work of Z. Ding was supported by the UK EPSRC under grant number EP/P009719/2 and by H2020-MSCA-RISE-2015 under grant number 690750.

\section{REFERENCES}

[1] C. I, et al, "New paradigm of 5G wireless Internet", IEEE Journal on Selected Areas in Commun., pp. 474-482, Vol. 34, No. 3, March 2016.

[2] H. Holma, "LTE small cell optimization: 3GPP evolution to Release 13," John Wiley \& Sons Ltd, 2016.

[3] F. Boccardi et al, "Five Disruptive Technology Directions for 5G," IEEE Communications Magazine, pp. 74-83, Feb. 2014.

[4] A. Hamza et al, "A survey on inter-cell interference coordination techniques in OFDMA-based cellular networks," IEEE Commun. Surveys \& Tutorials, pp. 1642- 1670, Vol.15, No.4, 2013.

[5] N. Miridakis, D. Vergados, "A survey on the successive interference cancellation performance for single-antenna and multiple-antenna OFDM systems," IEEE Commun. Surveys \& Tutorials, pp. 312-335, 2013.

[6] N. Saquib, E. Hossain, A. Kim, "Fractional frequency reuse for interference management in LTE-Advanced hetnets," IEEE Wireless Commun., pp. 113-122, April 2013.

[7] T. Norlan, R. Ganti, A. Ghosh, J. Andrews "Analytical evaluation of fractional frequency reuse for OFDMA cellular networks," IEEE Trans. Wireless Commun., Vol. 10, No. 12, pp. 4294-4305, 2011.
[8] J. He et al, "Analytical evaluation of higher order sectorization, frequency reuse and user classification methods in OFDMA networks," IEEE Trans. Wireless Commun., Vol. 15, No. 12, pp.8209 - 8222, Sept. 2016.

[9] Z. Zhang, Z. Ma, et al, "Full-Duplex Device-to-Device Aided Cooperative Non-Orthogonal Multiple Access," IEEE Trans. on Vehicular Tech. Aug. 2016.

[10] Z. Ding, F. Adachi, V. Poor, et al, "The application of MIMO to Nonorthogonal multiple access," IEEE Trans. Wireless Comm., Vol. 15 , No. 1, pp.537-552, Jan. 2016.

[11] Z. Ding and H. Poor, "Design of Massive-MIMO-NOMA With Limited Feedback," IEEE Signal Processing Lett., vol. 23, no. 5, pp. 629-633, May 2016.

[12] Z. Ding, et al, "A survey on non-orthogonal multiple access for 5G networks: Research challenges and future trends," IEEE Journal of Selected Areas in Comm., vol.35, no. 10, pp. 2181 - 2195, Oct. 2017.

[13] S. Weber and J. Andrews, "Transmission capacity of wireless networks," Foundations and Trends in Networking, 2012.

[14] X. Zhang, M. Haenggi, "On decoding the kth strongest user in Poisson networks with arbitrary fading distribution," In Proc. ACSSC'13, 2013.

[15] X. Zhang, M. Haenggi, 'The performance of successive interference cancellation in random wireless networks," IEEE Tran. Information Theory, Vol. 60, No. 10, pp. 6368 - 6388, July 2014.

[16] M. Wildemeersch et al, "Successive interference cancellation in uplink cellular networks," In Proc. SPAWC'13, 2013.

[17] G. Geraci, M. Wildemeersch, and T. Quek, "Energy Efficiency of Distributed Signal Processing in Wireless Networks: A Cross-Layer Analysis," IEEE Tran. Signal Processing, Vol. 64, No. 4, pp.1034-1047, Feb. 2016.

[18] B. Blaszczyszyn, H. Keeler, "Studying the SINR process of the typical user in Poisson networks using its factorial moment measures," IEEE Tran. Information Theory, pp.6774-6794, Dec. 2015.

[19] C. Ma et al, "On the performance of successive interference cancellation in D2D-enabled cellular networks," In Proc. IEEE INFOCOM'15, 2015.

[20] Mehlfuhrer et al, "The Vienna LTE simulators - Enabling reproducibility in wireless communications research," EURASIP Journal on Advances in Signal Processing, pp.1-14, 2011:29, 2011.

[21] L. Chen et al, "System-level simulation methodology and platform for mobile cellular systems," IEEE Communications Magazine, Vol. 49, No. 7, 2011.

[22] J. Andrews, F. Baccelli, and R. Ganti, "A tractable approach to coverage and rate in cellular networks," IEEE Transactions on Communications, Vol. 59, No. 11, pp. 3122-3134, Nov. 2011.

[23] J. He et al, "Statistical model of OFDMA cellular networks uplink interference using Lognormal distribution," IEEE Wireless Communications Letters, Vol.2, No.5, pp. 575-578, Oct. 2013.

[24] T. Norlan et al, "Analytical modeling of uplink cellular networks," IEEE Trans. on Wireless Communications, pp.2669-2679, June 2013.

[25] A. Wyner, "Shannon-theoretic approach to a Gaussian cellular multiaccess channel," IEEE Transactions on Information Theory, Vol. 40, No. 6, pp.1713-1727, Nov. 1994.

[26] H. Haas and S. McLaughlin, "A derivation of the PDF of adjacent channel interference in a cellular system," IEEE Communications Letters, Vol. 8, No. 2, Feb. 2004.

[27] Y. Zhu et al, "Distribution of uplink inter-cell interference in OFDMA networks with power control," In Proc. IEEE ICC'14, 2014.

[28] S. Elayoubi and O. Haddada, "Uplink intercell interference and capacity in 3G LTE systems," In Proc. IEEE ICON'07, 2007.

[29] M. Karray, "Evaluation of the blocking probability and the throughput in the uplink of wireless cellular networks," In Proc. IEEE ComNet'10, 2010.

[30] H. Tabassum et al, "A framework for uplink intercell interference modeling with channel-based scheduling," IEEE Transactions on Wireless Communications, Vol. 12, No. 1, pp. 206-219, Jan. 2013.

[31] H. Tabassum et al, "A statistical model of uplink inter-cell interference with slow and fast power control mechanisms," IEEE Transactions on Communications, Vol. 61, No. 9, pp. 3953-3966, Sep. 2013.

[32] S. Singh et al, "Moment-matched lognormal modeling of uplink interference with power control and cell selection," IEEE Transactions on Wireless Communications, Vol. 9, No. 3, pp. 932-938, Mar. 2010.

[33] H. Tabassum et al, "A generic interference model for uplink OFDMA networks with fractional frequency reuse," IEEE Trans. Vehicular Tech., Vol. 63, No. 3, pp.1491-1497, Mar. 2014.

[34] H. Chang and I. Rubin, "Optimal downlink and uplink fractional frequency reuse in cellular wireless networks," IEEE Transactions on Vehicular Technology, April 2015. 
TABLE III

MEAN SITE THROUGHPUT AND GAIN OVER NON-SIC SETTING $\left(N_{\mathrm{STG}}=0\right)$.

\begin{tabular}{|c|c|c|c|c|c|c|c|c|c|}
\hline \multicolumn{10}{|c|}{ Mean site throughput (Mbps) and gains (\%) (for simulation results) or modelling deviation (\%) (for analytical model results) } \\
\hline & $N_{\text {stg }}$ & 0 & & 1 & & 2 & & 3 & \\
\hline$\epsilon$ & $\alpha / \beta$ & Sim. / Gain & Mod. / Dev. & Sim. / Gain & Mod. / Dev. & Sim. / Gain & Mod. / Dev. & Sim. / Gain & Mod. / Dev. \\
\hline \multirow[t]{4}{*}{$0 \mathrm{~dB}$} & $0.55 / 0.4$ & $13.61 / 0.00$ & $13.69 / 0.59$ & $15.58 / 14.52$ & $15.40 /-1.18$ & $15.73 / 15.62$ & $15.55 /-1.16$ & $15.82 / 16.25$ & $15.56 /-1.63$ \\
\hline & $0.55 / 1.0$ & $12.78 / 0.00$ & $12.82 / 0.28$ & $14.85 / 16.23$ & $14.70 /-1.03$ & $15.18 / 18.77$ & $14.87 /-2.03$ & $15.24 / 19.23$ & $14.89 /-2.32$ \\
\hline & $1.00 / 0.4$ & $12.69 / 0.00$ & $12.76 / 0.55$ & $15.12 / 19.14$ & $15.32 / 1.31$ & $15.42 / 21.51$ & $15.51 / 0.58$ & $15.41 / 21.42$ & $15.53 / 0.74$ \\
\hline & $1.00 / 1.0$ & $12.26 / 0.00$ & $12.09 /-1.36$ & $14.54 / 18.55$ & $14.76 / 1.53$ & $14.79 / 20.61$ & $14.97 / 1.25$ & $14.90 / 21.52$ & $14.99 / 0.62$ \\
\hline \multirow{4}{*}{$-2 \mathrm{~dB}$} & $0.55 / 0.4$ & $13.61 / 0.00$ & $13.69 / 0.59$ & $16.30 / 19.81$ & $16.37 / 0.41$ & $17.04 / 25.20$ & $17.03 /-0.06$ & $17.18 / 26.23$ & $17.14 /-0.22$ \\
\hline & $0.55 / 1.0$ & $12.78 / 0.00$ & $12.82 / 0.28$ & $15.62 / 22.23$ & $15.61 /-0.09$ & $16.40 / 28.35$ & $16.29 /-0.71$ & $16.58 / 29.70$ & $16.41 /-0.99$ \\
\hline & $1.00 / 0.4$ & $12.69 / 0.00$ & $12.76 / 0.55$ & $15.89 / 25.20$ & $16.25 / 2.26$ & $16.81 / 32.42$ & $17.02 / 1.24$ & $16.85 / 32.71$ & $17.14 / 1.74$ \\
\hline & $1.00 / 1.0$ & $12.26 / 0.00$ & $12.09 /-1.36$ & $15.25 / 24.40$ & $15.61 / 2.32$ & $16.16 / 31.76$ & $16.40 / 1.50$ & $16.31 / 33.01$ & $16.53 / 1.38$ \\
\hline \multirow[t]{4}{*}{$-4 \mathrm{~dB}$} & $0.55 / 0.4$ & $13.61 / 0.00$ & $13.69 / 0.59$ & $16.92 / 24.34$ & $17.12 / 1.16$ & $18.51 / 36.03$ & $18.65 / 0.76$ & $19.18 / 40.96$ & $19.25 / 0.38$ \\
\hline & $0.55 / 1.0$ & $12.78 / 0.00$ & $12.82 / 0.28$ & $16.22 / 26.88$ & $16.29 / 0.47$ & $17.87 / 39.78$ & $17.82 /-0.27$ & $18.65 / 45.91$ & $18.44 /-1.11$ \\
\hline & $1.00 / 0.4$ & $12.69 / 0.00$ & $12.76 / 0.55$ & $16.66 / 31.23$ & $16.81 / 0.89$ & $18.37 / 44.73$ & $18.28 /-0.51$ & $19.04 / 49.97$ & $18.74 /-1.55$ \\
\hline & $1.00 / 1.0$ & $12.26 / 0.00$ & $12.09 /-1.36$ & $16.00 / 30.45$ & $16.06 / 0.42$ & $17.70 / 44.32$ & $17.50 /-1.09$ & $18.53 / 51.08$ & $17.98 /-2.97$ \\
\hline \multirow[t]{4}{*}{$-8 \mathrm{~dB}$} & $0.55 / 0.4$ & $13.61 / 0.00$ & $13.69 / 0.59$ & $17.47 / 28.35$ & $17.54 / 0.40$ & $20.09 / 47.65$ & $19.99 /-0.49$ & $22.18 / 63.02$ & $21.96 /-1.02$ \\
\hline & $0.55 / 1.0$ & $12.78 / 0.00$ & $12.82 / 0.28$ & $16.70 / 30.65$ & $16.75 / 0.29$ & 19.34 / 51.35 & $19.16 /-0.96$ & 21.47 / 68.01 & $21.08 /-1.83$ \\
\hline & $1.00 / 0.4$ & $12.69 / 0.00$ & $12.76 / 0.55$ & 17.17 / 35.23 & $17.38 / 1.24$ & 20.04 / 57.84 & $19.99 /-0.21$ & 22.24 / 75.24 & $22.08 /-0.74$ \\
\hline & $1.00 / 1.0$ & $12.26 / 0.00$ & $12.09 /-1.36$ & $16.47 / 34.30$ & $16.65 / 1.09$ & $19.43 / 58.47$ & $19.18 /-1.31$ & $21.52 / 75.52$ & $21.20 /-1.51$ \\
\hline
\end{tabular}

[35] H. ElSawy and E. Hossain, "On stochastic geometry modeling of cellular uplink transmission with truncated channel inversion power control," IEEE Transactions on Wireless Communications, Vol. 13, No. 8, pp. 4454-4469, Aug. 2014.

[36] L. Wang et al, "An analytical framework for multi-layer partial frequency reuse scheme design in mobile communication systems," IEEE Transactions on Vehicular Technology, Nov. 2015.

[37] 3GPP TR 36.814 V9.0.0, "Further advancements for E-UTRA physical layer aspects, Technical Report, March 2010.

[38] D. Lopez-Perez, H. Claussen, L. Ho, "Improved frequency reuse schemes with horizontal sector offset for LTE," in Proc. of IEEE PIMRC'13, 2013.

[39] L. Fenton, "The sum of log-normal probability distributions in scatter transmission systems," IEEE Trans. Commun., Vol. 8, No. 1, pp. 57-67, March 1960.

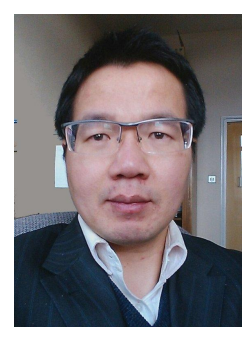

Jianhua He received his BSc and MSc degrees from Huazhong University of Science and Technology (HUST), China, and a PhD degree from Nanyang Technological University, Singapore, in 1995, 1998 and 2002, respectively. He joined HUST in 2001 as an Associate Professor. From 2004 to 2011, he has been with University of Bristol, University of Essex and University of Swansea. Dr He is a Lecturer at Aston University, UK. His main research interests are $5 \mathrm{G}$ networks, connected vehicles, autonomous driving, Internet of things, AI for OCR and wireless newtorks. He has authored or co-authored over 100 technical papers in major international journals and conferences. Dr He is the coordinator of EU Horizon2020 project COSAFE on connected autonomous vehicles. He is an IEEE Senior Member.

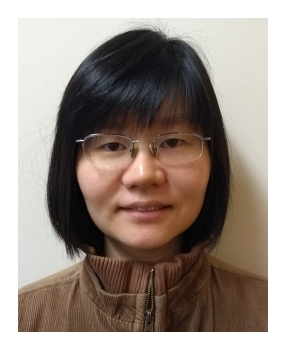

Zuoyin Tang is currently a Lecturer in the School of Engineering and Applied Science, Aston University, UK. She obtained her PhD degree from University of Bath, UK, in 2008. She has authored and coauthored over 40 technical papers in major international journals and conferences. Dr Tang's main research interests include resource management for cellular networks, Internet of things and wireless sensor networks.

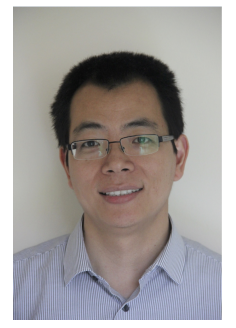

Zhiguo Ding (S'03-M'05) received his B.Eng in Electrical Engineering from the Beijing University of Posts and Telecommunications in 2000, and the $\mathrm{Ph} . \mathrm{D}$ degree in Electrical Engineering from Imperial College London in 2005. From Jul. 2005 to Apr. 2018, he was working in Queen's University Belfast, Imperial College, Newcastle University and Lancaster University. Since Apr. 2018, he has been with the University of Manchester as a Professor in Communications. From Oct. 2012 to Sept. 2018, he has also been an academic visitor in Princeton University. Dr Ding' research interests are 5G networks, game theory, cooperative and energy harvesting networks and statistical signal processing. He is serving as an Editor for IEEE Transactions on Communications, IEEE Transactions on Vehicular Technology, and Journal of Wireless Communications and Mobile Computing, and was an Editor for IEEE Wireless Communication Letters, IEEE Communication Letters from 2013 to 2016. He received the best paper award in IET ICWMC-2009 and IEEE WCSP-2014, the EU Marie Curie Fellowship 2012-2014, the Top IEEE TVT Editor 2017, IEEE Heinrich Hertz Award 2018 and the IEEE Jack Neubauer Memorial Award 2018.

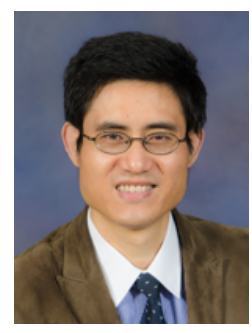

Dapeng Wu (S'98-M'04-SM06-F'13) received a B.E. degree in electrical engineering from Huazhong University of Science and Technology, Wuhan, China, in 1990, an M.E. degree in electrical engineering from Beijing University of Posts and Telecommunications, Beijing, China, in 1997, and a $\mathrm{Ph} . \mathrm{D}$. degree in electrical and computer engineering from Carnegie Mellon University, Pittsburgh, PA, in 2003. He is a professor at the Department of Electrical and Computer Engineering, University of Florida, Gainesville, FL. His research interests are in the areas of networking, communications, signal processing, computer vision, machine learning, smart grid, and information and network security. Currently, he serves as Editor in Chief of IEEE Transactions on Network Science and Engineering, and Associate Editor of IEEE Transactions on Communications, IEEE Transactions on Signal and Information Processing over Networks, and IEEE Signal Processing Magazine. He was the founding Editor-in-Chief of Journal of Advances in Multimedia between 2006 and 2008, and an Associate Editor for IEEE Transactions on Circuits and Systems for Video Technology, IEEE Transactions on Wireless Communications and IEEE Transactions on Vehicular Technology. He is also a guest-editor for IEEE Journal on Selected Areas in Communications (JSAC), Special Issue on Cross-layer Optimized Wireless Multimedia Communications. He is an IEEE Fellow. 